\title{
A histamine gene expression program in human macrophages.
}

Shahan Mamoor, MS1

1Thomas Jefferson School of Law

San Diego, CA 92901

mamoorsk@tjsl.edu

The significance of histamine signaling in septic shock is debated (1-5). The macrophage is one of the critical cell types that provide rapid immune responses to early events in septic shock (6-11). To understand in a systematic and unbiased manner the transcriptional behavior of macrophage in response to the immunologic effector histamine, we used a public dataset (12) to describe the most significant changes in the gene expression program of human primary macrophages in response to histamine exposure. We found that of the genes whose expression was most different between macrophages exposed to histamine and the naïve macrophage there were a selection of genes that could be assigned to 22 discrete modules, including genes encoding enzymes involved in glycosylation, the mitochondria and metabolism, the quality control and translation of proteins, the ER stress response, the Rab family of molecules, genes that bind or encode molecules involved in genetic and epigenetic modulation of DNA and RNA, CXCL5, glutamate signaling, and the cyclin dependent kinase CDK14. It appears that macrophages respond to histamine exposure by altering gene expression at a relatively large number of loci but by a relatively small order of magnitude.

Keywords: septic shock, sepsis, macrophages, histamine, differential gene expression. 


\section{Introduction}

Septic shock, a consequence of sepsis (an infection of the bloodstream), is a leading killer of adults in the United States with over 200,000 deaths per year resulting in patients diagnosed with sepsis (13). The in-hospital mortality rate from septic shock is estimated to be between 14-29\%, depending on the criteria used to diagnose patients (14); other estimates indicate a $10-20 \%$ mortality rate from sepsis and a $40-80 \%$ mortality rate from septic shock (13). A key issue in sepsis is a biochemical imbalance of oxygen demand and oxygen delivery throughout organ systems, leading to shock (15). Treatments are targeted towards resolving certain parameters such as arterial lactate, venous oxygen, and blood $\mathrm{pH}$, as well as preventing fatal clotting complications such as disseminated intravascular coagulation, resolving the triggering factor (pathogen), and other supportive measures such as maintaining sufficient blood pressure with fluid resuscitation and ventilation, and can include the use of glucocorticoids (15-20).

Histamine is a key chemical effector in the immune system (21). It drives allergic responses in tissues such as the skin and the in the respiratory system (22), and it has effects on the nervous system (23). Thus far, studies have debated the importance of histamine signaling in septic shock. While one study showed histamine release in patients with sepsis (1), another argued that concentrations of histamine were not elevated in patients with septic shock (2). In an experimental sepsis model, activation of the Histamine H3 receptor leads to depression of cardiac function (3). In a porcine model of septic shock, histamine blockade lead to increased survival time (4). Another study argued that endogenous histamine played an essential role in promoting tissue damage associated with septic shock (5). Together, these data argue that histamine signaling may be an important contributing mechanism in the pathogenesis of sepsis and septic shock.

Macrophages are a key cell type in the innate immune system and they play an important role in the pathogenesis of septic shock as they differentiate from monocytes in the blood stream to macrophages in tissues (6-11). Levels of macrophage inhibitory factor are 
increased in the plasma of patients with severe sepsis (7), and neutralization of macrophage inhibitory factor in a cecal ligation and puncture (CLP) murine model of sepsis prevented peritonitis that results in death (8). Another study using the CLP model demonstrated that delivery of bone marrow stromal cells prior to or after the time of puncture lead to a statistically significant improvement in survival, showed that this was dependent on IL-10, and argued that this survival benefit conferred by injection of bone marrow stromal cells was the result of increased IL-10 production by macrophages, induced in macrophages via prostaglandin-E2 signaling from stromal cell to macrophage (11). Together, these evidence argue that the macrophage is an important cell type in the disease process associated with sepsis and septic shock.

In this study, we used a public dataset (12) to perform a systems-level analysis of transcription in macrophages following histamine exposure. We determined, on a global level, the genes whose expression was most different between naive macrophages isolated from human donors and those treated with histamine. We found twenty-two different modules of genes that were differentially expressed following exposure to histamine with an emphasis on genes involved in the glycosylation of cellular substrates, in metabolism, in the function of the mitochondria, in vesicular trafficking, in protein synthesis and quality control, and of the machinery that repairs, replicates, and modifies DNA and RNA. Included among the most differentially expressed genes were a series of non-coding RNA transcripts.

\section{Methods}

GSE66015 was utilized for this analysis in conjunction with GEO2R. The investigators that acquired the data contained with GSE66015 utilized Illumina HumanHT-12 V4.0 expression beadchip microarray technology on primary macrophage isolated from two human donors, with and without 8 hours of histamine exposure at $0.5 \mathrm{mg} / \mathrm{mL}$ (12). In determination of rank of global differential expression, for the non-treated controls, an additional mock-treated macrophage sample was included for the non-treated macrophage control group; the mRNA 
expression values for this sample (essentially a vehicle control) were not included in this analysis (GEO2R provides data regarding rank of differential expression for all genes in the transcriptome, but the exact mRNA expression values for only the 250 most differentially expressed genes in the transcriptome). For our analysis here, the "Benjamini and Hochberg" method of $p$-value adjustment was used. Log transformation was set to "Auto-detect" and the submitter supplied category of platform annotation was used. The statistical significance of each gene presented here with respect to it being differentially expressed relative to all of the genes whose products compose the transcriptome was calculated by GEO2R. We conducted a separate statistical test to determine whether the mean RNA expression levels of each differentially expressed gene changed to a statistically significant degree with and without histamine exposure (and not relative to the rest of the transcriptome) using an unpaired, twotailed t-test with Welch's correction by PRISM (8.1.2) (227). The results of this separate statistical test, which does not alter the fact that each of these genes is statistically significantly differentially expressed transcriptome-wide, is presented with a corresponding $p$-value on each graph comparing gene expression levels between histamine-treated and naïve macrophage groups.

\section{Results}

We compared the transcriptomes of primary human macrophages from two donors (12), in the naive state and following treatment with histamine for 8 hours, to globally define the transcriptional behavior of macrophages in response to histamine. We classified a subset of the top $1 \%$ of the most differentially expressed genes in macrophages following histamine exposure into 22 categories of genes.

Epigenetics: Histamine activates gene expression of the lysine methyltransferases KMT5B and KMT5C, as well as other enzymes involved in epigenetic modification of DNA. 
KMT5B was among the most differentially expressed genes in the transcriptome of histamine-treated primary macrophages from humans when compared to naive macrophages from the same donor (Table 1). It was ranked 117 out of 47323 transcripts detected and measured, with respect to differential expression, or the magnitude of change in expression in macrophages treated or not with histamine (Table 1; $p=0.00956)$. The expression of KMT5B in macrophages treated with histamine was significantly higher than in naive macrophages (Figure $1 ; p=0.0121)$.

KMT5B was surprisingly also among the most differentially expressed genes in the transcriptome of histamine-treated primary macrophages from humans when compared to naive macrophages from the same donor (Table 1). It was ranked 82 out of 47323 transcripts detected and measured, with respect to differential expression (Table 1; $p=0.00956)$. The expression of KMT5C in macrophages treated with histamine was significantly higher than in naive macrophages (Figure $1 ; p=0.0069$ ).

PCGF6 was also among the most differentially expressed genes in the transcriptome of histamine-treated primary macrophages from humans when compared to naive macrophages from the same donor, ranking 22 out of 47323 transcripts detected globally (Table 1; $p=$ ). The expression of PCGF6 was significantly lower in macrophages treated with histamine than in naïve macrophages (Figure $1 ; p=0.0063$ ).

DOT1L was also one of the most differentially expressed genes in the transcriptome of histamine-treated primary macrophages from humans when compared to naive macrophages from the same donor, ranking 96 out of 47323 transcripts detected globally (Table $1 ; p=$ ). The expression of DOT1L was significantly lower in macrophages treated with histamine than in naïve macrophages (Figure 1; $p=0.019216$ ).

Immunity: Histamine induces the gene expression of CXCL5 while decreasing IL-12B expression. 
CXCL5 was the most differentially expressed genes in the transcriptome of histaminetreated primary macrophages from humans when compared to naive macrophages from the same donor, out of all 47323 transcripts detected globally (Table 1; $p=0.000114$ ). The expression of CXCL5 was significantly higher in macrophages treated with histamine than in naïve macrophages (Figure 2; $p=0.0364$ ).

IL-12B was among the most differentially expressed genes in the transcriptome of histamine-treated primary macrophages from humans when compared to naive macrophages from the same donor, ranking 62 out of 47323 transcripts detected globally (Table 1; $\mathrm{p}=0.014346)$. The expression of IL-12B was significantly lower in macrophages treated with histamine than in naïve macrophages (Figure $2 ; p=0.0035$ ).

Immunity: Genes whose products function in immune defense, including a complement cascade member, are perturbed following histamine treatment of macrophages.

C1QTNF3 was among the most differentially expressed genes in the transcriptome of histamine-treated primary macrophages from humans when compared to naive macrophages from the same donor, ranking 229 out of 47323 transcripts detected globally (Table 1; $\mathrm{p}=0.016968$ ). The expression of C1QTNF3 was lower in macrophages treated with histamine than in naïve macrophages, and this difference approached statistical significance (Figure 3; $p=0.0515)$.

CTSL was also among the most differentially expressed genes in the transcriptome of histamine-treated primary macrophages from humans when compared to naive macrophages from the same donor, ranking 47 out of 47323 transcripts detected globally (Table 1; $p=$ ). The expression of CTSL was significantly higher in macrophages treated with histamine than in naïve macrophages (Figure $4 ; p=0.0110$ ).

DEFB4A was also among the most differentially expressed genes in the transcriptome of histamine-treated primary macrophages from humans when compared to naive macrophages from the same donor, ranking 66 out of 47323 transcripts detected globally 
(Table $1 ; p=0.006489$ ). The expression of DEFB4A was significantly lower in macrophages treated with histamine than in naïve macrophages (Figure 4; $\mathrm{p}=0.0019$ ).

TRIL was also among the most differentially expressed genes in the transcriptome of histamine-treated primary macrophages from humans when compared to naive macrophages from the same donor, ranking 97 out of 47323 transcripts detected globally (Table 1; $p=$ ). The expression of TRIL was lower in macrophages treated with histamine than in naïve macrophages (Figure 4; $p=0.018916)$.

Non-coding RNA: The expression of a host of non-coding RNA including microRNAs and long intergenic non-coding is induced by histamine.

miR1470 was among the most differentially expressed genes in the transcriptome of histamine-treated primary macrophages from humans when compared to naive macrophages from the same donor, ranking 184 out of 47323 transcripts detected globally (Table 1;

$\mathrm{p}=0.018779$ ). The expression of miR1470 was significantly lower in macrophages treated with histamine than in naïve macrophages (Figure $5 ; p=0.0035$ ).

miR192 was also among the most differentially expressed genes in the transcriptome of histamine-treated primary macrophages from humans when compared to naive macrophages from the same donor, ranking 226 out of 47323 transcripts detected globally (Table 1; $\mathrm{p}=0.01642)$. The expression of miR192 was significantly higher in macrophages treated with histamine than in naïve macrophages (Figure $5 ; p=0.0014$ ).

miR202 was also among the most differentially expressed genes in the transcriptome of histamine-treated primary macrophages from humans when compared to naive macrophages from the same donor, ranking 232 out of 47323 transcripts detected globally (Table 1; $\mathrm{p}=0.016609$ ). The expression of miR202 was significantly lower in macrophages treated with histamine than in naïve macrophages (Figure 5; $p=0.0035$ ). 
NRAV was also among the most differentially expressed genes in the transcriptome of histamine-treated primary macrophages from humans when compared to naive macrophages from the same donor, ranking 231 out of 47323 transcripts detected globally (Table 1; $\mathrm{p}=0.016572$ ). The expression of NRAV was significantly higher in macrophages treated with histamine than in naïve macrophages (Figure 5; $p=0.0145$ ).

LINC00853 was also among the most differentially expressed genes in the transcriptome of histamine-treated primary macrophages from humans when compared to naive macrophages from the same donor, ranking 65 out of 47323 transcripts detected globally (Table 1; $p=0.012136$ ). The expression of LINC00853 was significantly higher in macrophages treated with histamine than in naïve macrophages (Figure $5 ; \mathrm{p}<0.0001$ ).

LINC00473 was also among the most differentially expressed genes in the transcriptome of histamine-treated primary macrophages from humans when compared to naive macrophages from the same donor, ranking 102 out of 47323 transcripts detected globally (Table 1; $\mathrm{p}=0.019936)$. The expression of LINC00473 was higher in macrophages treated with histamine than in naïve macrophages (Figure 5; $p=0.0849$ ).

SNORD54 was also among the most differentially expressed genes in the transcriptome of histamine-treated primary macrophages from humans when compared to naive macrophages from the same donor, ranking 3 out of 47323 transcripts detected globally (Table $1 ; \mathrm{p}=0.002262)$. The expression of SNORD54 was significantly lower in macrophages treated with histamine than in naïve macrophages (Figure $5 ; p=0.0260$ ).

RPL21P28 was also among the most differentially expressed genes in the transcriptome of histamine-treated primary macrophages from humans when compared to naive macrophages from the same donor, ranking 36 out of 47323 transcripts detected globally (Table 1; $p=0.005581$ ). The expression of RPL21P28 was lower in macrophages treated with histamine than in naïve macrophages (Figure $5 ; \mathrm{p}=0.0608$ ).

ST13P4 was also among the most differentially expressed genes in the transcriptome of histamine-treated primary macrophages from humans when compared to naive macrophages 
from the same donor, ranking 143 out of 47323 transcripts detected globally (Table $1 ; p=$ ). The expression of ST13P4 was significantly lower in macrophages treated with histamine than in naïve macrophages (Figure 5; $p=0.0150$ ).

DNA/RNA Machines: Levels of RNA Polymerase POLR2F increases while levels of POLR3H increase in macrophages.

POLR2F was among the most differentially expressed genes in the transcriptome of histamine-treated primary macrophages from humans when compared to naive macrophages from the same donor, ranking 214 out of 47323 transcripts detected globally (Table 1;

$\mathrm{p}=0.015583)$. The expression of POLR2F was significantly higher in macrophages treated with histamine than in naïve macrophages (Figure 6; $p=0.0051$ ).

POLR3H was also among the most differentially expressed genes in the transcriptome of histamine-treated primary macrophages from humans when compared to naive macrophages from the same donor, ranking 153 out of 47323 transcripts detected globally (Table 1; $\mathrm{p}=0.012151)$. The expression of POLR3H was significantly lower in macrophages treated with histamine than in naïve macrophages (Figure $6 ; p=0.0028$ ).

The RAB Family: Histamine activates gene expression of RAB6B and RAB20 as well as two effectors in the RAB family.

RAB20 was among the most differentially expressed genes in the transcriptome of histamine-treated primary macrophages from humans when compared to naive macrophages from the same donor, ranking 48 out of 47323 transcripts detected globally (Table $1 ; p=$ ). The expression of RAB20 was significantly higher in macrophages treated with histamine than in naïve macrophages (Figure 7; $p=0.0235$ ).

RAB6B was also among the most differentially expressed genes in the transcriptome of histamine-treated primary macrophages from humans when compared to naive macrophages from the same donor, ranking 142 out of 47323 transcripts detected globally (Table 1; 
$\mathrm{p}=0.011439$ ). The expression of RAB6B was higher in macrophages treated with histamine than in naïve macrophages (Figure $7 ; p=0.1937$ ).

RIN3 was also among the most differentially expressed genes in the transcriptome of histamine-treated primary macrophages from humans when compared to naive macrophages from the same donor, ranking 10 out of 47323 transcripts detected globally (Table $1 ; p=$ 0.00715). The expression of RIN3 was significantly higher in macrophages treated with histamine than in naïve macrophages (Figure $7 ; p=0.0304$ ).

$\mathrm{CHM}$ was also among the most differentially expressed genes in the transcriptome of histamine-treated primary macrophages from humans when compared to naive macrophages from the same donor, ranking 2 out of 47323 transcripts detected globally (Table 1;

$\mathrm{p}=0.000102)$. The expression of $\mathrm{CHM}$ was higher in macrophages treated with histamine than in naïve macrophages, and this difference in gene expression approached the level of the statistical significance (Figure $7 ; \mathrm{p}=0.0556$ ).

The Cell Cycle: CDK14 and Cyclin $\mathrm{H}$ are transcriptionally activated following histamine exposure in macrophages.

CDK14 was among the most differentially expressed genes in the transcriptome of histamine-treated primary macrophages from humans when compared to naive macrophages from the same donor, ranking 4 out of 47323 transcripts detected globally (Table 1; $\mathrm{p}=0.001533)$. The expression of CDK14 was significantly higher in macrophages treated with histamine than in naïve macrophages (Figure 8; $p=0.0136$ ).

Cyclin $\mathrm{H}(\mathrm{CCNH})$ was also among the most differentially expressed genes in the transcriptome of histamine-treated primary macrophages from humans when compared to naive macrophages from the same donor, ranking 157 out of 47323 transcripts detected globally (Table 1; $\mathrm{p}=0.012397$ ). The expression of $\mathrm{Cyclin} \mathrm{H}$ was significantly higher in macrophages treated with histamine than in naïve macrophages (Figure $8 ; p=0.0121$ ). 
Glycosylation: Multiple enzymes involved in glycosylation are differentially expressed by macrophages following histamine exposure.

B3GALNT was among the most differentially expressed genes in the transcriptome of histamine-treated primary macrophages from humans when compared to naive macrophages from the same donor, ranking 41 out of 47323 transcripts detected globally (Table $1 ; p=$ 0.010162). The expression of B3GALNT was significantly lower in macrophages treated with histamine than in naïve macrophages (Figure 9; $p=0.0015$ ).

FUCA2 was also among the most differentially expressed genes in the transcriptome of histamine-treated primary macrophages from humans when compared to naive macrophages from the same donor, ranking 6 out of 47323 transcripts detected globally (Table 1; $\mathrm{p}=0.0099564)$. The expression of FUCA2 was significantly lower in macrophages treated with histamine than in naïve macrophages (Figure 9; $p=0.0004$ ).

UGGT2 was among the most differentially expressed genes in the transcriptome of histamine-treated primary macrophages from humans when compared to naive macrophages from the same donor, ranking 150 out of 47323 transcripts detected globally (Table 1; $\mathrm{p}=0.012016)$. The expression of UGGT2 was lower in macrophages treated with histamine than in naïve macrophages (Figure 9; $p=0.0718$ ).

MGAT1 was also among the most differentially expressed genes in the transcriptome of histamine-treated primary macrophages from humans when compared to naive macrophages from the same donor, ranking 7 out of 47323 transcripts detected globally (Table 1; $\mathrm{p}=0.00358$ ). The expression of MGAT1 was significantly higher in macrophages treated with histamine than in naïve macrophages (Figure 9; $p=0.0024)$.

GALNT16 was also among the most differentially expressed genes in the transcriptome of histamine-treated primary macrophages from humans when compared to naive macrophages from the same donor, ranking 167 out of 47323 transcripts detected globally (Table 1; $\mathrm{p}=$ ). The expression of GALNT16 was lower in macrophages treated with histamine than in naïve macrophages (Figure 9; $p=0.01153$ ). 
LGALS9C was also among the most differentially expressed genes in the transcriptome of histamine-treated primary macrophages from humans when compared to naive macrophages from the same donor, ranking 190 out of 47323 transcripts detected globally (Table 1; $\mathrm{p}=$ ). The expression of LGALS9C was significantly lower in macrophages treated with histamine than in naïve macrophages (Figure 9; $p=0.0169$ ).

UAP1L1 was also among the most differentially expressed genes in the transcriptome of histamine-treated primary macrophages from humans when compared to naive macrophages from the same donor, ranking 197 out of 47323 transcripts detected globally (Table 1; $\mathrm{p}=0.014881$ ). The expression of UAP1L1 was higher in macrophages treated with histamine than in naïve macrophages (Figure 9; $p=.1044$ ).

ST6GALNAC3 was also among the most differentially expressed genes in the transcriptome of histamine-treated primary macrophages from humans when compared to naive macrophages from the same donor, ranking 230 out of 47323 transcripts detected globally (Table 1; $\mathrm{p}=0.016569$ ). The expression of ST6GALNAC3 was significantly higher in macrophages treated with histamine than in naïve macrophages (Figure 9; $p=0.0025$ ).

The Mitochondria: Genes of the mitochondria are perturbed by histamine exposure.

MRPL47 was among the most differentially expressed genes in the transcriptome of histamine-treated primary macrophages from humans when compared to naive macrophages from the same donor, ranking 21 out of 47323 transcripts detected globally (Table 1; $\mathrm{p}=0.005928$ ). The expression of MRPL47 was significantly lower in macrophages treated with histamine than in naïve macrophages (Figure 10; $p=0.0038$ ).

MRPL55 was also among the most differentially expressed genes in the transcriptome of histamine-treated primary macrophages from humans when compared to naive macrophages from the same donor, ranking 100 out of 47323 transcripts detected globally (Table 1; $\mathrm{p}=0.011721$ ). The expression of MRPL55 was higher in macrophages treated with histamine than in naïve macrophages (Figure 10; $p=0.0654$ ). 
SUCLG1 was also among the most differentially expressed genes in the transcriptome of histamine-treated primary macrophages from humans when compared to naive macrophages from the same donor, ranking 0.0037 out of 47323 transcripts detected globally (Table 1; $\mathrm{p}=0.016339$ ). The expression of SUCLG1 was significantly lower in macrophages treated with histamine than in naïve macrophages (Figure 10; $p=0.0037$ ).

CIAO1 was also among the most differentially expressed genes in the transcriptome of histamine-treated primary macrophages from humans when compared to naive macrophages from the same donor, ranking 33 out of 47323 transcripts detected globally (Table 1; $p=$ ). The expression of CIAO1 was higher in macrophages treated with histamine than in naïve macrophages (Figure 10; $p=0.003758$ ).

TSPO was also among the most differentially expressed genes in the transcriptome of histamine-treated primary macrophages from humans when compared to naive macrophages from the same donor, ranking 9 out of 47323 transcripts detected globally (Table 1; $\mathrm{p}=0.001645)$. The expression of TSPO was lower in macrophages treated with histamine than in naïve macrophages (Figure 10; $p=0.0772$ ).

ROMO1 was also among the most differentially expressed genes in the transcriptome of histamine-treated primary macrophages from humans when compared to naive macrophages from the same donor, ranking 121 out of 47323 transcripts detected globally (Table 1; $\mathrm{p}=0.009892$ ). The expression of ROMO1 was significantly higher in macrophages treated with histamine than in naïve macrophages (Figure 10; $p=0.0142$ ).

COX7B was also among the most differentially expressed genes in the transcriptome of histamine-treated primary macrophages from humans when compared to naive macrophages from the same donor, ranking 212 out of 47323 transcripts detected globally (Table 1; $\mathrm{p}=0.017049$ ). The expression of COX7B was significantly lower in macrophages treated with histamine than in naïve macrophages (Figure 10; $p=0.0273$ ).

PC was also among the most differentially expressed genes in the transcriptome of histamine-treated primary macrophages from humans when compared to naive macrophages 
from the same donor, ranking 216 out of 47323 transcripts detected globally (Table 1 ; $\mathrm{p}=0.015602)$. The expression of $\mathrm{PC}$ was significantly higher in macrophages treated with histamine than in naïve macrophages (Figure 10; $p=0.0382$ ).

NOX1 was also among the most differentially expressed genes in the transcriptome of histamine-treated primary macrophages from humans when compared to naive macrophages from the same donor, ranking 179 out of 47323 transcripts detected globally (Table 1; $\mathrm{p}=0.018344)$. The expression of NOX1 was significantly lower in macrophages treated with histamine than in naïve macrophages (Figure 10; $p=0.0228$ ).

Transcription Factors; The expression of multiple transcription factors is activated by histamine in macrophages, but Tox3 expression decreases.

BCL11A was among the most differentially expressed genes in the transcriptome of histamine-treated primary macrophages from humans when compared to naive macrophages from the same donor, ranking 14 out of 47323 transcripts detected globally (Table 1; $p=0.002242)$. The expression of BCL11A was significantly higher in macrophages treated with histamine than in naïve macrophages (Figure 11; $p=0.0067$ ).

VEZF1 was also among the most differentially expressed genes in the transcriptome of histamine-treated primary macrophages from humans when compared to naive macrophages from the same donor, ranking 23 out of 47323 transcripts detected globally (Table 1; $p=$ 0.006334). The expression of VEZF1 was higher in macrophages treated with histamine than in naïve macrophages (Figure 11; $p=0.0785$ ).

TOX3 was also among the most differentially expressed genes in the transcriptome of histamine-treated primary macrophages from humans when compared to naive macrophages from the same donor, ranking 198 out of 47323 transcripts detected globally (Table 1; $p=0.01493)$. The expression of TOX3 was significantly lower in macrophages treated with histamine than in naïve macrophages (Figure $11 ; \mathrm{p}=0.0084$ ). 
PAXBP1 was also among the most differentially expressed genes in the transcriptome of histamine-treated primary macrophages from humans when compared to naive macrophages from the same donor, ranking 54 out of 47323 transcripts detected globally (Table $1 ; p=0.0116355$ ). The expression of PAXBP1 was higher in macrophages treated with histamine than in naïve macrophages (Figure 11; $p=0.0588$ ).

MAZ was also among the most differentially expressed genes in the transcriptome of histamine-treated primary macrophages from humans when compared to naive macrophages from the same donor, ranking 78 out of 47323 transcripts detected globally (Table 1; $p=0.016488$ ). The expression of MAZ was lower in macrophages treated with histamine than in naïve macrophages (Figure $11 ; p=.1037$ ).

FHL3 was also among the most differentially expressed genes in the transcriptome of histamine-treated primary macrophages from humans when compared to naive macrophages from the same donor, ranking 38 out of 47323 transcripts detected globally (Table 1; $p=0.004255)$. The expression of FHL3 was significantly lower in macrophages treated with histamine than in naïve macrophages (Figure 11; $p=0.0024$ ).

DNA and RNA Machines: The expression of machinery involved in DNA replication and repair is affected by histamine exposure in macrophages.

MCM2 was among the most differentially expressed genes in the transcriptome of histamine-treated primary macrophages from humans when compared to naive macrophages from the same donor, ranking 89 out of 47323 transcripts detected globally (Table 1; $\mathrm{p}=0.04418$ ). The expression of MCM2 was lower in macrophages treated with histamine than in naïve macrophages (Figure 12; $p=0.1055$ ).

ENPP4 was also among the most differentially expressed genes in the transcriptome of histamine-treated primary macrophages from humans when compared to naive macrophages from the same donor, ranking 46 out of 47323 transcripts detected globally (Table 1; 
$\mathrm{p}=0.00212)$. The expression of ENPP4 was significantly higher in macrophages treated with histamine than in naïve macrophages (Figure 12; $p=0.0185$ ).

RRN3P1 was also among the most differentially expressed genes in the transcriptome of histamine-treated primary macrophages from humans when compared to naive macrophages from the same donor, ranking 8 out of 47323 transcripts detected globally (Table 1; $\mathrm{p}=0.00174)$. The expression of RRN3P1 was higher in macrophages treated with histamine than in naïve macrophages, and this difference in expression approached the level of statistical significance (Figure 12; $p=0.001174$ ).

APEX2 was also among the most differentially expressed genes in the transcriptome of histamine-treated primary macrophages from humans when compared to naive macrophages from the same donor, ranking 125 out of 47323 transcripts detected globally (Table 1; $\mathrm{p}=0.010012)$. The expression of APEX2 was higher in macrophages treated with histamine than in naïve macrophages (Figure 12; $p=0.0623$ ).

PABPC1 was also among the most differentially expressed genes in the transcriptome of histamine-treated primary macrophages from humans when compared to naive macrophages from the same donor, ranking 110 out of 47323 transcripts detected globally (Table 1; $\mathrm{p}=0.019873$ ). The expression of PABPC1 was significantly higher in macrophages treated with histamine than in naïve macrophages (Figure 12; $\mathrm{p}=0.0107$ ).

TDP1 was also among the most differentially expressed genes in the transcriptome of histamine-treated primary macrophages from humans when compared to naive macrophages from the same donor, ranking 101 out of 47323 transcripts detected globally (Table 1; $\mathrm{p}=0.008733$ ). The expression of TDP1 was significantly lower in macrophages treated with histamine than in naïve macrophages (Figure 12; $p=0.0102$ ).

HNRNPK was also among the most differentially expressed genes in the transcriptome of histamine-treated primary macrophages from humans when compared to naive macrophages from the same donor, ranking 61 out of 47323 transcripts detected globally 
(Table 1; $\mathrm{p}=0.0098$ ). The expression of HNRNPK was significantly lower in macrophages treated with histamine than in naïve macrophages (Figure 12; $p=0.00602$ ).

HNRNPCL1 was also among the most differentially expressed genes in the transcriptome of histamine-treated primary macrophages from humans when compared to naive macrophages from the same donor, ranking 154 out of 47323 transcripts detected globally (Table 1; $\mathrm{p}=0.012178$ ). The expression of HNRNPCL1 was higher in macrophages treated with histamine than in naïve macrophages (Figure 12; $\mathrm{p}=0.1342$ ).

Cellular Transport: Vesicular trafficking gene expression is affected by histamine exposure.

GAPVD1 was among the most differentially expressed genes in the transcriptome of histamine-treated primary macrophages from humans when compared to naive macrophages from the same donor, ranking 25 out of 47323 transcripts detected globally (Table 1; $p=$ 0.003454). The expression of GAPVD1 was significantly higher in macrophages treated with histamine than in naïve macrophages (Figure 13; $p=0.0017$ ).

AP2S1 was also among the most differentially expressed genes in the transcriptome of histamine-treated primary macrophages, ranking 220 out of 47323 transcripts detected globally (Table 1; $p=0.016057$ ). The expression of AP2S1 was significantly lower in macrophages treated with histamine than in naïve macrophages (Figure 13; $p=0.0061$ ).

WDFY1 was also among the most differentially expressed genes in the transcriptome of histamine-treated primary macrophages, ranking 183 out of 47323 transcripts detected globally (Table 1; $p=0.013491$ ). The expression of WDFY1 was higher in macrophages treated with histamine than in naïve macrophages (Figure 13; $p=0.2328$ ).

VAMP7 was also among the most differentially expressed genes in the transcriptome of histamine-treated primary macrophages, ranking 139 out of 47323 transcripts detected globally (Table $1 ; \mathrm{p}=0.011184$ ). The expression of VAMP7 was higher in macrophages treated with histamine than in naïve macrophages (Figure 13; $p=0.0511$ ). 
SEC22B was also among the most differentially expressed genes in the transcriptome of histamine-treated primary macrophages, ranking 19 out of 47323 transcripts detected globally (Table 1; $\mathrm{p}=0.002742$ ). The expression of SEC22B was higher in macrophages treated with histamine than in naïve macrophages (Figure 13; $p=0.1192$ ).

HPS3 was also among the most differentially expressed genes in the transcriptome of histamine-treated primary macrophages, ranking 239 out of 47323 transcripts detected globally (Table $1 ; p=0.016941$ ). The expression of HPS3 was significantly higher in macrophages treated with histamine than in naïve macrophages (Figure 13; $p=0.0045$ ).

SNF8 was also among the most differentially expressed genes in the transcriptome of histamine-treated primary macrophages, ranking 51 out of 47323 transcripts detected globally (Table 1; $\mathrm{p}=0.0052$ ). The expression of SNF8 was significantly lower in macrophages treated with histamine than in naïve macrophages (Figure 13; $p=0.0054$ ).

CLN8 was also among the most differentially expressed genes in the transcriptome of histamine-treated primary macrophages from humans when compared to naive macrophages from the same donor, ranking 84 out of 47323 transcripts detected globally (Table 1; $\mathrm{p}=0.005613)$. The expression of CLN8 was significantly higher in macrophages treated with histamine than in naïve macrophages (Figure 13; $p=0.0113$ ).

KTN1 was also among the most differentially expressed genes in the transcriptome of histamine-treated primary macrophages from humans when compared to naive macrophages from the same donor, ranking 187 out of 47323 transcripts detected globally (Table $1 ; p=$ ). The expression of KTN1 was significantly lower in macrophages treated with histamine than in naïve macrophages (Figure 13; $p=0.0061$ ).

TRAK1 was also among the most differentially expressed genes in the transcriptome of histamine-treated primary macrophages from humans when compared to naive macrophages from the same donor, ranking 35 out of 47323 transcripts detected globally (Table 1; $\mathrm{p}=0.004008)$. The expression of TRAK1 was significantly higher in macrophages treated with histamine than in naïve macrophages (Figure 12; $p=0.0390$ ). 
Ubiquitin: Multiple genes involved in ubiquitin modification are perturbed by histamine treatment in macrophages.

JOSD1 was among the most differentially expressed genes in the transcriptome of histamine-treated primary macrophages from humans when compared to naive macrophages from the same donor, ranking 140 out of 47323 transcripts detected globally (Table 1; $\mathrm{p}=0.011204)$. The expression of JOSD1 was higher in macrophages treated with histamine than in naïve macrophages (Figure 14; $\mathrm{p}=0.0606$ ).

BTBD1 was also among the most differentially expressed genes in the transcriptome of histamine-treated primary macrophages, ranking 222 out of 47323 transcripts detected globally (Table 1; $p=0.016189$ ). The expression of BTBD1 was significantly lower in macrophages treated with histamine than in naïve macrophages (Figure 14; $p=0.0159$ ).

RNF40 was also among the most differentially expressed genes in the transcriptome of histamine-treated primary macrophages, ranking 90 out of 47323 transcripts detected globally (Table 1; $\mathrm{p}=0.008143$ ). The expression of RNF40 was significantly higher in macrophages treated with histamine than in naïve macrophages (Figure 14; $p=0.0076$ ).

TRIM14 was also among the most differentially expressed genes in the transcriptome of histamine-treated primary macrophages, ranking 163 out of 47323 transcripts detected globally (Table 1; $\mathrm{p}=0.0126212$ ). The expression of TRIM14 was significantly higher in macrophages treated with histamine than in naïve macrophages (Figure 14; $p=0.0009$ ).

TRIM49D1 was also among the most differentially expressed genes in the transcriptome of histamine-treated primary macrophages from humans when compared to naive macrophages from the same donor, ranking 146 out of 47323 transcripts detected globally (Table 1; $\mathrm{p}=0.011904$ ). The expression of TRIM49D1 was higher in macrophages treated with histamine than in naïve macrophages (Figure 14; $p=0.0901$ ).

USP4 was also among the most differentially expressed genes in the transcriptome of histamine-treated primary macrophages from humans when compared to naive macrophages from the same donor, ranking 136 out of 47323 transcripts detected globally (Table 1; 
$\mathrm{p}=0.010741)$. The expression of USP4 was significantly higher in macrophages treated with histamine than in naïve macrophages (Figure 14; $p=0.0117$ ).

Phosphatases and Kinases: The gene expression of specific phosphatases and kinases is affected by histamine exposure.

PPME1 was among the most differentially expressed genes in the transcriptome of histamine-treated primary macrophages from humans when compared to naive macrophages from the same donor, ranking 50 out of 47323 transcripts detected globally (Table 1; $p=0.005127)$. The expression of PPME1 was significantly lower in macrophages treated with histamine than in naïve macrophages (Figure 15; $p=0.0086$ ).

PPP2R3A was also among the most differentially expressed genes in the transcriptome of histamine-treated primary macrophages from humans when compared to naive macrophages from the same donor, ranking 177 out of 47323 transcripts detected globally (Table 1; $\mathrm{p}=0.018847$ ). The expression of PPP2R3A was higher in macrophages treated with histamine than in naïve macrophages (Figure 15; $p=0.0673$ ).

PRKRA was also among the most differentially expressed genes in the transcriptome of histamine-treated primary macrophages from humans when compared to naive macrophages from the same donor, ranking 103 out of 47323 transcripts detected globally (Table 1; $p=0.019492)$. The expression of PRKRA was significantly lower in macrophages treated with histamine than in naïve macrophages (Figure 15; $p=0.0338$ ).

ABL2 was also among the most differentially expressed genes in the transcriptome of histamine-treated primary macrophages from humans when compared to naive macrophages from the same donor, ranking 126 out of 47323 transcripts detected globally (Table 1; $p=0.010594)$. The expression of ABL2 was significantly higher in macrophages treated with histamine than in naïve macrophages (Figure 15; $p=0.0278$ ).

WNK1 was also among the most differentially expressed genes in the transcriptome of histamine-treated primary macrophages from humans when compared to naive macrophages 
from the same donor, ranking 131 out of 47323 transcripts detected globally (Table 1; $p=$ 0.010595). The expression of WNK1 was significantly higher in macrophages treated with histamine than in naïve macrophages (Figure 15; $p=0.0037$ ).

Calcium Signaling: Gene expression of molecules involved in calcium signaling is affected by histamine exposure.

CASR was among the most differentially expressed genes in the transcriptome of histamine-treated primary macrophages from humans when compared to naive macrophages from the same donor, ranking 194 out of 47323 transcripts detected globally (Table 1; $p=0.014501)$. The expression of CASR was significantly lower in macrophages treated with histamine than in naïve macrophages (Figure 16; $p=0.0088$ ).

CALD1 was also among the most differentially expressed genes in the transcriptome of histamine-treated primary macrophages from humans when compared to naive macrophages from the same donor, ranking 186 out of 47323 transcripts detected globally (Table 1; $\mathrm{p}=0.013846)$. The expression of CALD1 was significantly lower in macrophages treated with histamine than in naïve macrophages (Figure 16; $p=0.0296$ ).

$\mathrm{SRL}$ was also among the most differentially expressed genes in the transcriptome of histamine-treated primary macrophages from humans when compared to naive macrophages from the same donor, ranking 168 out of 47323 transcripts detected globally (Table 1; $p=0.012902)$. The expression of $S R L$ was higher in macrophages treated with histamine than in naïve macrophages (Figure 16; $p=0.0744$ ).

\section{Membrane Biochemistry: Enzymes involved in manipulation of cellular phospholipids are} affected at the level of gene expression by histamine.

PIK3CG was among the most differentially expressed genes in the transcriptome of histamine-treated primary macrophages from humans when compared to naive macrophages 
from the same donor, ranking 59 out of 47323 transcripts detected globally (Table 1; $\mathrm{p}=0.005867$ ). The expression of PIK3CG was higher in macrophages treated with histamine than in naïve macrophages (Figure $17 ; p=0.0994$ ).

GDPD1 was also among the most differentially expressed genes in the transcriptome of histamine-treated primary macrophages from humans when compared to naive macrophages from the same donor, ranking 196 out of 47323 transcripts detected globally (Table 1; $p=0.014693$ ). The expression of GDPD1 was higher in macrophages treated with histamine than in naïve macrophages (Figure $17 ; \mathrm{p}=0.1790$ ).

PIP4K2C was also among the most differentially expressed genes in the transcriptome of histamine-treated primary macrophages from humans when compared to naive macrophages from the same donor, ranking 228 out of 47323 transcripts detected globally (Table 1; $\mathrm{p}=0.01652$ ). The expression of PIP4K2C was significantly higher in macrophages treated with histamine than in naïve macrophages (Figure 17; $p=0.0293$ ).

Translation and the ER Stress Response: Histamine modulates the expression of genes involved in protein translation and quality control of proteins / the ER stress response.

UTP14C was among the most differentially expressed genes in the transcriptome of histamine-treated primary macrophages from humans when compared to naive macrophages from the same donor, ranking 238 out of 47323 transcripts detected globally (Table 1; $\mathrm{p}=0.0169$ ). The expression of UTP14C was significantly higher in macrophages treated with histamine than in naïve macrophages (Figure 18; $p=0.0197$ ).

EIF6 was also among the most differentially expressed genes in the transcriptome of histamine-treated primary macrophages from humans when compared to naive macrophages from the same donor, ranking 86 out of 47323 transcripts detected globally (Table 1; $\mathrm{p}=0.016505)$. The expression of EIF6 was lower in macrophages treated with histamine than in naïve macrophages (Figure 18; $p=0.0856$ ). 
ADAT2 was also among the most differentially expressed genes in the transcriptome of histamine-treated primary macrophages from humans when compared to naive macrophages from the same donor, ranking 156 out of 47323 transcripts detected globally (Table 1; $\mathrm{p}=0.018456)$. The expression of ADAT2 was higher in macrophages treated with histamine than in naïve macrophages (Figure 18; $p=0.1054)$.

CGGBP1 was also among the most differentially expressed genes in the transcriptome of histamine-treated primary macrophages from humans when compared to naive macrophages from the same donor, ranking 71 out of 47323 transcripts detected globally (Table 1; $\mathrm{p}=0.006679$ ). The expression of CGGBP1 was higher in macrophages treated with histamine than in naïve macrophages (Figure 18; $p=0.1329$ ).

FKBP3 was also among the most differentially expressed genes in the transcriptome of histamine-treated primary macrophages from humans when compared to naive macrophages from the same donor, ranking 118 out of 47323 transcripts detected globally (Table 1; $\mathrm{p}=0.009578)$. The expression of FKBP3 was lower in macrophages treated with histamine than in naïve macrophages (Figure 18; $p=0.2687$ ).

EDEM3 was also among the most differentially expressed genes in the transcriptome of histamine-treated primary macrophages from humans when compared to naive macrophages from the same donor, ranking 53 out of 47323 transcripts detected globally (Table 1; $\mathrm{p}=0.005227)$. The expression of EDEM3 was significantly lower in macrophages treated with histamine than in naïve macrophages (Figure 18; $p<0.0001$ ).

EDEM1 was also among the most differentially expressed genes in the transcriptome of histamine-treated primary macrophages from humans when compared to naive macrophages from the same donor, ranking 58 out of 47323 transcripts detected globally (Table 1; $\mathrm{p}=0.005774)$. The expression of EDEM1 was higher in macrophages treated with histamine than in naïve macrophages, and this difference in expression approached the level of statistical significance (Figure 18; $p=0.0562$ ). 
The Nervous System: Genes typically expressed in or canonically associated with the brain and the nervous system are affected by histamine exposure in macrophages.

PCDH11Y was among the most differentially expressed genes in the transcriptome of histamine-treated primary macrophages from humans when compared to naive macrophages from the same donor, ranking 161 out of 47323 transcripts detected globally (Table $1 ; p=$ 0.012565). The expression of PCDH11Y was significantly higher in macrophages treated with histamine than in naïve macrophages (Figure 19; $p=0.0073$ ).

OCLM was also among the most differentially expressed genes in the transcriptome of histamine-treated primary macrophages, ranking 88 out of 47323 transcripts detected globally (Table 1; $\mathrm{p}=0.007772$ ). The expression of OCLM was significantly higher in macrophages treated with histamine than in naïve macrophages (Figure 19; $\mathrm{p}=0.0157$ ).

MRGPRD was also among the most differentially expressed genes in the transcriptome of histamine-treated primary macrophages, ranking 69 out of 47323 transcripts detected globally (Table $1 ; p=0.006557$ ). The expression of MRGPRD was higher in macrophages treated with histamine than in naïve macrophages, and this difference in expression approached the level of statistical significance (Figure 19; $p=0.0534$ ).

IgLON5 was also among the most differentially expressed genes in the transcriptome of histamine-treated primary macrophages, ranking 52 out of 47323 transcripts detected globally (Table $1 ; p=0.01024$ ). The expression of IgLON5 was significantly lower in macrophages treated with histamine than in naïve macrophages (Figure 19; $\mathrm{p}=0.0023$ ).

ENC1 was also among the most differentially expressed genes in the transcriptome of histamine-treated primary macrophages, ranking 18 out of 47323 transcripts detected globally (Table 1; $\mathrm{p}=0.001728$ ). The expression of ENC1 was significantly lower in macrophages treated with histamine than in naïve macrophages (Figure 19; $p=0.0037$ ).

NRGN was also among the most differentially expressed genes in the transcriptome of histamine-treated primary macrophages from humans when compared to naive macrophages from the same donor, ranking 26 out of 47323 transcripts detected globally (Table 1; 
$p=0.000306)$. The expression of NRGN was significantly lower in macrophages treated with histamine than in naïve macrophages (Figure 19; $\mathrm{p}=0.0065$ ).

NGEF was also among the most differentially expressed genes in the transcriptome of histamine-treated primary macrophages from humans when compared to naive macrophages from the same donor, ranking 104 out of 47323 transcripts detected globally (Table 1; $\mathrm{p}=0.01018$ ). The expression of NGEF was significantly higher in macrophages treated with histamine than in naïve macrophages (Figure 19; $p=0.0010$ ).

The Nuclear Envelope : Nup37 and other genes at the nuclear envelope are affected by histamine exposure.

Nup37 was among the most differentially expressed genes in the transcriptome of histamine-treated primary macrophages from humans when compared to naive macrophages from the same donor, ranking 123 out of 47323 transcripts detected globally (Table 1; $\mathrm{p}=0.009947)$. The expression of Nup37 was significantly higher in macrophages treated with histamine than in naïve macrophages (Figure 20; $p=0.0422$ ).

LMNA was also among the most differentially expressed genes in the transcriptome of histamine-treated primary macrophages from humans when compared to naive macrophages from the same donor, ranking 249 out of 47323 transcripts detected globally (Table 1; $\mathrm{p}=0.017635)$. The expression of LMNA was higher in macrophages treated with histamine than in naïve macrophages (Figure 20; $p=0.0855$ ).

SYNE1 was also among the most differentially expressed genes in the transcriptome of histamine-treated primary macrophages from humans when compared to naive macrophages from the same donor, ranking 180 out of 47323 transcripts detected globally (Table 1; $p=0.013414)$. The expression of SYNE1 was significantly lower in macrophages treated with histamine than in naïve macrophages (Figure 20; $p=0.0396$ ).

The Cytoskeleton: Cytoskeletal genes are affected by histamine treatment in macrophages. 
TTC17 was among the most differentially expressed genes in the transcriptome of histamine-treated primary macrophages from humans when compared to naive macrophages from the same donor, ranking 120 out of 47323 transcripts detected globally (Table 1; $\mathrm{p}=0.009779$ ). The expression of TTC17 was significantly lower in macrophages treated with histamine than in naïve macrophages (Figure $21 ; p=0.0107$ ).

SPC25 was also among the most differentially expressed genes in the transcriptome of histamine-treated primary macrophages from humans when compared to naive macrophages from the same donor, ranking 124 out of 47323 transcripts detected globally (Table $1 ; p=$ 0.009987). The expression of SPC25 was significantly lower in macrophages treated with histamine than in naïve macrophages (Figure $21 ; p=0.0054$ ).

SRFBP1 was also among the most differentially expressed genes in the transcriptome of histamine-treated primary macrophages from humans when compared to naive macrophages from the same donor, ranking 112 out of 47323 transcripts detected globally (Table 1; $\mathrm{p}=0.009202$ ). The expression of SRFBP1 was lower in macrophages treated with histamine than in naïve macrophages (Figure $21 ; p=0.0585$ ).

Metabolism: A network of genes that function in cellular metabolism are differentially expressed following histamine treatment of macrophages.

OSBPL8 was among the most differentially expressed genes in the transcriptome of histamine-treated primary macrophages from humans when compared to naive macrophages from the same donor, ranking 15 out of 47323 transcripts detected globally (Table 1; $\mathrm{p}=0.002398)$. The expression of OSBPL8 was significantly higher in macrophages treated with histamine than in naïve macrophages (Figure 22; $p=0.0373$ ).

$\mathrm{FAH}$ was also among the most differentially expressed genes in the transcriptome of histamine-treated primary macrophages from humans when compared to naive macrophages from the same donor, ranking 203 out of 47323 transcripts detected globally (Table 1; 
$\mathrm{p}=0.005075)$. The expression of $\mathrm{FAH}$ was significantly higher in macrophages treated with histamine than in naïve macrophages (Figure 22; $\mathrm{p}=0.0179$ ).

$\mathrm{RDH} 11$ was also among the most differentially expressed genes in the transcriptome of histamine-treated primary macrophages from humans when compared to naive macrophages from the same donor, ranking 144 out of 47323 transcripts detected globally (Table 1; $\mathrm{p}=0.012096)$. The expression of $\mathrm{RDH} 11$ was significantly lower in macrophages treated with histamine than in naïve macrophages (Figure 22; $p=0.0220$ ).

PTGR2 was also among the most differentially expressed genes in the transcriptome of histamine-treated primary macrophages from humans when compared to naive macrophages from the same donor, ranking 128 out of 47323 transcripts detected globally (Table 1; $\mathrm{p}=0.010254)$. The expression of PTGR2 was significantly higher in macrophages treated with histamine than in naïve macrophages (Figure 22; $p=0.0142$ ).

MVD was also among the most differentially expressed genes in the transcriptome of histamine-treated primary macrophages from humans when compared to naive macrophages from the same donor, ranking 91 out of 47323 transcripts detected globally (Table 1; $\mathrm{p}=0.019732)$. The expression of MVD was significantly lower in macrophages treated with histamine than in naïve macrophages (Figure 22; $p=0.0288$ ).

LPA was also among the most differentially expressed genes in the transcriptome of histamine-treated primary macrophages from humans when compared to naive macrophages from the same donor, ranking 241 out of 47323 transcripts detected globally (Table 1; $\mathrm{p}=0.017156)$. The expression of LPA was significantly higher in macrophages treated with histamine than in naïve macrophages (Figure 22; $p=0.0019$ ).

DHODH was also among the most differentially expressed genes in the transcriptome of histamine-treated primary macrophages, ranking 247 out of 47323 transcripts detected globally (Table $1 ; p=0.017501)$. The expression of $\mathrm{DHODH}$ was significantly lower in macrophages treated with histamine than in naïve macrophages (Figure 22; $p=0.0201$ ). 
ABHD17A was also among the most differentially expressed genes in the transcriptome of histamine-treated primary macrophages, ranking 37 out of 47323 transcripts detected globally (Table $1 ; p=0.006213$ ). The expression of ABHD17A was significantly lower in macrophages treated with histamine than in naïve macrophages (Figure 22; $p=0.0035$ ).

SUOX was also among the most differentially expressed genes in the transcriptome of histamine-treated primary macrophages, ranking 55 out of 47323 transcripts detected globally (Table 1; $\mathrm{p}=0.014304)$. The expression of SUOX was higher in macrophages treated with histamine than in naïve macrophages (Figure 22; $p=0.0790$ ).

LRP11 was also among the most differentially expressed genes in the transcriptome of histamine-treated primary macrophages, ranking 93 out of 47323 transcripts detected globally (Table 1; $p=0.008343$ ). The expression of LRP11 was lower in macrophages treated with histamine than in naïve macrophages (Figure 22; $p=0.1173$ ).

GLUD2 was also among the most differentially expressed genes in the transcriptome of histamine-treated primary macrophages, ranking 31 out of 47323 transcripts detected globally (Table $1 ; p=0.009457)$. The expression of GLUD2 was significantly higher in macrophages treated with histamine than in naïve macrophages (Figure 22; $p=0.0163$ ).

SLC1A3 was also among the most differentially expressed genes in the transcriptome of histamine-treated primary macrophages from humans when compared to naive macrophages from the same donor, ranking 5 out of 47323 transcripts detected globally; this was statistically quite significant (Table $1 ; p=0.001118$ ). The expression of SLC1A3 was higher in macrophages treated with histamine than in naïve macrophages (Figure 22; $p=0.1089$ ).

Survival: Genes that function in survival are differentially expressed after macrophage exposure to histamine.

TC2N was among the most differentially expressed genes in the transcriptome of histamine-treated primary macrophages from humans when compared to naive macrophages from the same donor, ranking 74 out of 47323 transcripts detected globally (Table 1; 
$\mathrm{p}=0.006794)$. The expression of TC2N was significantly lower in macrophages treated with histamine than in naïve macrophages (Figure 23; $p=0.0044$ ).

PMAIP1 (NOXA) was also among the most differentially expressed genes in the transcriptome of histamine-treated primary macrophages from humans when compared to naive macrophages from the same donor, ranking 13 out of 47323 transcripts detected globally (Table 1; $\mathrm{p}=0.001918$ ). The expression of PMAIP1/NOXA was lower in macrophages treated with histamine than in naïve macrophages (Figure 23; $p=0.0881$ ).

The Skin: Genes that have structural roles in the skin are differentially expressed following histamine exposure in macrophages

KRTAP10-12 was among the most differentially expressed genes in the transcriptome of histamine-treated primary macrophages from humans when compared to naive macrophages from the same donor, ranking 221 out of 47323 transcripts detected globally (Table 1; $\mathrm{p}=0.016108)$. The expression of KRTAP10-12 was significantly lower in macrophages treated with histamine than in naïve macrophages (Figure 24; $p=0.0045$ ).

KRT39 was also among the most differentially expressed genes in the transcriptome of histamine-treated primary macrophages from humans when compared to naive macrophages from the same donor, ranking 176 out of 47323 transcripts detected globally (Table 1; $\mathrm{p}=0.013306)$. The expression of KRT39 was higher in macrophages treated with histamine than in naïve macrophages (Figure 24; $p=0.1543$ ).

DSC3 was also among the most differentially expressed genes in the transcriptome of histamine-treated primary macrophages from humans when compared to naive macrophages from the same donor, ranking 130 out of 47323 transcripts detected globally (Table $1 ; p=$ 0.010533). The expression of DSC3 was significantly higher in macrophages treated with histamine than in naïve macrophages (Figure 24; $p=0.0057$ ).

\section{Discussion}


Septic shock is a leading killer of children and adults (13), resulting in more than 200,000 deaths per year, and there is literature to suggest that antagonism of histamine may be beneficial in septic shock (4). In this study, we mined a public dataset (12) to perform a transcriptome-wide analysis of the macrophage response to histamine. By assessing macrophage transcription at the systems level, and in primary cells from humans, we found that the genes that were most differentially expressed, or whose gene expression changed the most significantly from the naïve to histamine-treated states, could be classified into 22 broad categories which reflect key cellular processes, pathways or structures.

We found that CXCL5 was, from over 47,000 transcripts, the gene whose expression changed the most after histamine expression. The level of CXCL5 increased significantly after exposure to histamine. CXCL5 has been shown to increase levels of CXCL1 and CXCL2 in the plasma, and increased CXCL1 and CXCL2 lead to a compromised ability to form a chemokine gradient (24). Disruption of the chemokine gradient as a result of CXCL5-mediated increases in CXCL1 and CXCL2 in this study lead to deficits in neutrophil trafficking and clearance of bacteria (24). In the same study, using a separate LPS-inhalation model, the investigators found that CXCL5 mediated the influx of neutrophils in to the lung during associated inflammatory respones. Another study showed that CXCL5 derived from enterocytes in the gut could regulate the levels of IL-17 and G-CSF, and was important in neutrophil homeostasis through CXCR2 (25).

Polymorphisms in IL12B are associated with the risk of developing multiple autoimmune and infectious diseases, including type I diabetes, asthma, psoriasis, and cerebral malaria (26-29). In humans, IL12 deficiency reflects itself as a compromised immune system, as one patient with a sequenced loss-of-function deletion mutation in IL12B suffered from infections caused by Salmonella enteritidis and bacille Calmette-Guérin (30). We found that exposure to histamine resulted in a significant decrease in the amount of IL-12B message present in macrophages. 
We also detected involvement of the glutamate metabolic pathway as being affected in human macrophages following histamine exposure. The fifth most differentially expressed gene in human macrophages exposed to histamine was SLC1A3, a solute carrier that functions in transporting glutamate across cell membranes. Moreover, GLUD2, glutamate dehydrogenase, which converts glutamate to alpha-ketoglutarate and ammonia, and whose expression and activity is thought to be controlled by the need of the cell for ATP, was also among the genes most differentially expressed by human macrophages following histamine exposure (31). Thus, genes which connect cellular demand for ATP and the metabolism of glutamate are among those whose expression is most different in histamine-treated macrophages from humans.

IL-12B and CXCL5 are just 2 out of over 100 genes presented here as a description of the transcriptional response to histamine in macrophages, demonstrating that histamine exerts an influence on macrophages that effects the expression of a wide variety of genes. However, the magnitude of the change in expression of these genes, so large that it was considered greater than the change in expression caused by histamine in $99 \%$ of the rest of the transcriptome, is small enough that in many cases the difference between expression of that gene in control and histamine-treated conditions was not statistically significant. These data indicate that histamine affects the expression of many genes in the transcriptome but that these changes are relatively modest. Histamine blockade in at least one model of septic shock has conferred a benefit to the organism (4). Studies should assess in different models the effect of pharmacological histamine blockade on sepsis and septic shock outcomes. 


\section{References}

1. Neugebauer, E., Lorenz, W., Rixen, D., Stinner, B., Sauer, S. and Dietz, W., 1996. Histamine release in sepsis: A prospective, controlled, clinical study|. Critical care medicine, 24(10), pp.1670-1677.

2. Jacobs, R.U.T.H., Kaliner, M.I.C.H.A.E.L., Shelhamer, J.H. and Parrillo, J.E., 1989. Blood histamine concentrations are not elevated in humans with septic shock. Critical care medicine, 17(1), pp.30-35.

3. Li, X., Eschun, G., Bose, D., Jacobs, H., Yang, J.J., Light, R.B. and Mink, S.N., 1998. Histamine $\mathrm{H} 3$ activation depresses cardiac function in experimental sepsis. Journal of Applied Physiology, 85(5), pp.1693-1701.

4. Byrne, K.A.R.L., Sielaff, T.D., Michna, B.A.R.B.A.R.A., Carey, P.D., Blocher, C.R., Vasquez, A.L.B.E.R.T.O. and Sugerman, H.J., 1990. Increased survival time after delayed histamine and prostaglandin blockade in a porcine model of severe sepsis-induced lung injury. Critical care medicine, 18(3), pp.303-308.

5. Hattori, M., Yamazaki, M., Ohashi, W., Tanaka, S., Hattori, K., Todoroki, K., Fujimori, T., Ohtsu, H., Matsuda, N. and Hattori, Y., 2016. Critical role of endogenous histamine in promoting end-organ tissue injury in sepsis. Intensive care medicine experimental, 4(1), p. 36.

6. Lehmann, L., Novender, U., Schroeder, S., Pietsch, T., von Spiegel, T., Putensen, C., Hoeft, A. and Stüber, F., 2001. Plasma levels of macrophage migration inhibitory factor are elevated in patients with severe sepsis. Intensive care medicine, 27(8), pp.1412-1415.

7. Calandra, T., Echtenacher, B., Le Roy, D., Pugin, J., Metz, C.N., Hültner, L., Heumann, D., Männel, D., Bucala, R. and Glauser, M.P., 2000. Protection from septic shock by neutralization of macrophage migration inhibitory factor. Nature medicine, 6(2), pp.164-170.

8. Gomez-Jimenez, J., Martin, M.C., Sauri, R., Segura, R.M., Esteban, F., Ruiz, J.C., Nuvials, X., Boveda, J.L., Peracaula, R. and Salgado, A., 1995. Interleukin-10 and the monocyte/ macrophage-induced inflammatory response in septic shock. Journal of Infectious Diseases, 171(2), pp.472-475.

9. Filkins, J.P., 1985, February. Monokines and the metabolic pathophysiology of septic shock. In Federation proceedings (Vol. 44, No. 2, pp. 300-304).

10. Welbourn, C.R.B. and Young, Y., 1992. Endotoxin, septic shock and acute lung injury: neutrophils, macrophages and inflammatory mediators. British journal of surgery, 79(10), pp.998-1003. 
11. Németh, K., Leelahavanichkul, A., Yuen, P.S., Mayer, B., Parmelee, A., Doi, K., Robey, P.G., Leelahavanichkul, K., Koller, B.H., Brown, J.M. and Hu, X., 2009. Bone marrow stromal cells attenuate sepsis via prostaglandin E 2-dependent reprogramming of host macrophages to increase their interleukin-10 production. Nature medicine, 15(1), p.42.

12. GSE66015. Anastasina M, Kainov D. University of Helsinki, Institute for Molecular Medicine, Finland.

13. Martin, G.S., 2012. Sepsis, severe sepsis and septic shock: changes in incidence, pathogens and outcomes. Expert review of anti-infective therapy, 10(6), pp.701-706.

14. Sterling, S.A., Puskarich, M.A., Glass, A.F., Guirgis, F. and Jones, A.E., 2017. The impact of the Sepsis-3 septic shock definition on previously defined septic shock patients. Critical care medicine, 45(9), p.1436.

15. Rivers, E., Nguyen, B., Havstad, S., Ressler, J., Muzzin, A., Knoblich, B., Peterson, E. and Tomlanovich, M., 2001. Early goal-directed therapy in the treatment of severe sepsis and septic shock. New England Journal of Medicine, 345(19), pp.1368-1377.

16. Annane, D., Sébille, V., Charpentier, C., Bollaert, P.E., François, B., Korach, J.M., Capellier, G., Cohen, Y., Azoulay, E., Troché, G. and Chaumet-Riffaut, P., 2002. Effect of treatment with low doses of hydrocortisone and fludrocortisone on mortality in patients with septic shock. Jama, 288(7), pp.862-871.

17. Rackow, E.C. and Astiz, M.E., 1991. Pathophysiology and treatment of septic shock. Jama, 266(4), pp.548-554.

18. Ledingham, I.M. and McArdle, C.S., 1978. Prospective study of the treatment of septic shock. The Lancet, 311(8075), pp.1194-1197.

19. Howell, M.D. and Davis, A.M., 2017. Management of sepsis and septic shock. Jama, 317(8), pp.847-848.

20. Keeley, A., Hine, P. and Nsutebu, E., 2017. The recognition and management of sepsis and septic shock: a guide for non-intensivists. Postgraduate medical journal, 93(1104), pp. 626-634.

21. Jutel, M., Watanabe, T., Akdis, M., Blaser, K. and Akdis, C.A., 2002. Immune regulation by histamine. Current opinion in immunology, 14(6), pp.735-740.

22. Cockcroft, D.W., Murdock, K.Y., Kirby, J. and Hargreave, F., 1987. Prediction of airway responsiveness to allergen from skin sensitivity to allergen and airway responsiveness to histamine. American Review of Respiratory Disease, 135(1), pp.264-267. 
23. Haas, H.L., Sergeeva, O.A. and Selbach, O., 2008. Histamine in the nervous system. Physiological reviews, 88(3), pp.1183-1241.

24. Mei, J., Liu, Y., Dai, N., Favara, M., Greene, T., Jeyaseelan, S., Poncz, M., Lee, J.S. and Worthen, G.S., 2010. CXCL5 regulates chemokine scavenging and pulmonary host defense to bacterial infection. Immunity, 33(1), pp.106-117.

25. Mei, J., Liu, Y., Dai, N., Hoffmann, C., Hudock, K.M., Zhang, P., Guttentag, S.H., Kolls, J.K., Oliver, P.M., Bushman, F.D. and Worthen, G.S., 2012. Cxcr2 and Cxcl5 regulate the IL-17/ G-CSF axis and neutrophil homeostasis in mice. The Journal of clinical investigation, 122(3), pp.974-986.

26. Morahan, G., Huang, D., Ymer, S.I., Cancilla, M.R., Stephen, K., Dabadghao, P., Werther, G., Tait, B.D., Harrison, L.C. and Colman, P.G., 2001. Linkage disequilibrium of a type 1 diabetes susceptibility locus with a regulatory IL12B allele. Nature genetics, 27(2), pp. 218-221.

27. Nair, R.P., Ruether, A., Stuart, P.E., Jenisch, S., Tejasvi, T., Hiremagalore, R., Schreiber, S., Kabelitz, D., Lim, H.W., Voorhees, J.J. and Christophers, E., 2008. Polymorphisms of the IL12B and IL23R genes are associated with psoriasis. Journal of Investigative Dermatology, 128(7), pp.1653-1661.

28. Randolph, A.G., Lange, C., Silverman, E.K., Lazarus, R., Silverman, E.S., Raby, B., Brown, A., Ozonoff, A., Richter, B. and Weiss, S.T., 2004. The IL12B gene is associated with asthma. The American Journal of Human Genetics, 75(4), pp.709-715.

29. Marquet, S., Doumbo, O., Cabantous, S., Poudiougou, B., Argiro, L., Safeukui, I., Konate, S., Sissoko, S., Chevereau, E., Traore, A. and Keita, M.M., 2008. A functional promoter variant in IL12B predisposes to cerebral malaria. Human Molecular Genetics, 17(14), pp. 2190-2195.

30. Picard, C., Fieschi, C., Altare, F., Al-Jumaah, S., Al-Hajjar, S., Feinberg, J., Dupuis, S., Soudais, C., Al-Mohsen, I.Z., Génin, E. and Lammas, D., 2002. Inherited interleukin-12 deficiency: IL12B genotype and clinical phenotype of 13 patients from six kindreds. The American Journal of Human Genetics, 70(2), pp.336-348.

31. Spanaki, C., Zaganas, I., Kleopa, K.A. and Plaitakis, A., 2010. Human GLUD2 glutamate dehydrogenase is expressed in neural and testicular supporting cells. Journal of Biological Chemistry, 285(22), pp.16748-16756. 

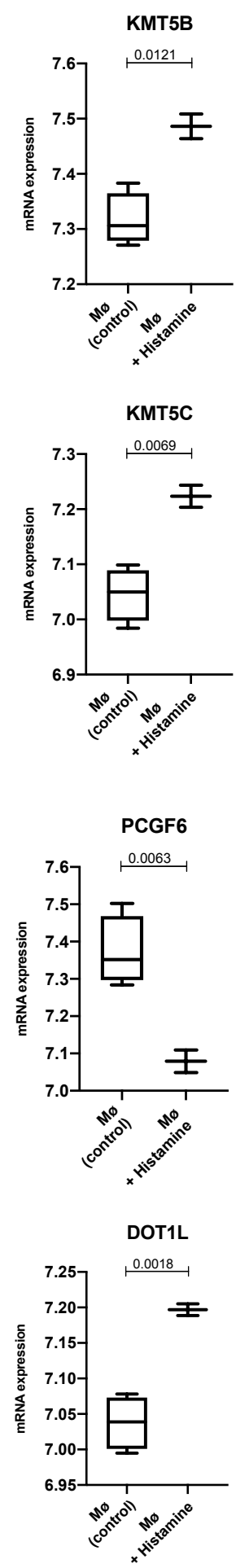
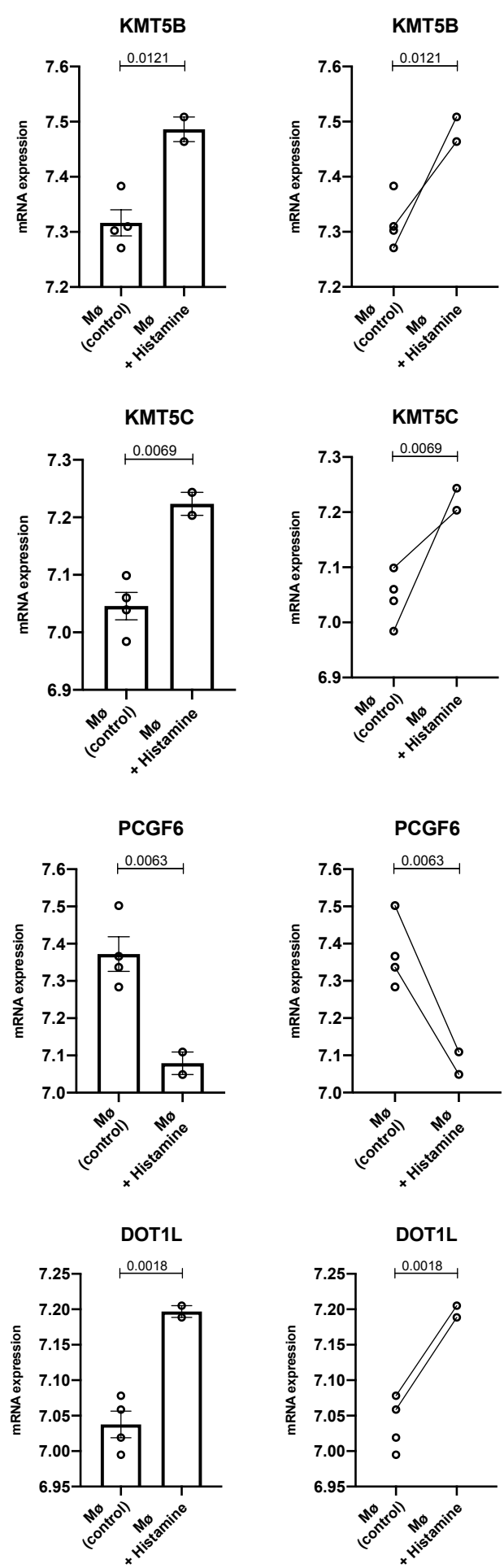

Figure 1: Epigenetics: Histamine activates gene expression of the lysine methyltransferases KMT5B and $\mathrm{KMT5C}$, as well as perturbing the expression of other enzymes involved in epigenetic modification of DNA

The expression of the indicated genes is graphically represented by box and whisker plot from minimum to maximum value (left), by bar graph (middle) with the error bars denoting the standard error of the mean, and by symbols and connecting lines (right). For control samples, the two connecting samples on the right hand panel represent macrophages from Donor $A$ and Donor $B$ with and without histamine treatment. There are two additional control samples, unconnected, representing mock treatment and mock histamine treatment. A statistical test was performed to determine if the difference in mRNA expression values between control and histamine-treated samples was significant; the result of this t-test is shown on each graph as a pvalue. 
CXCL5

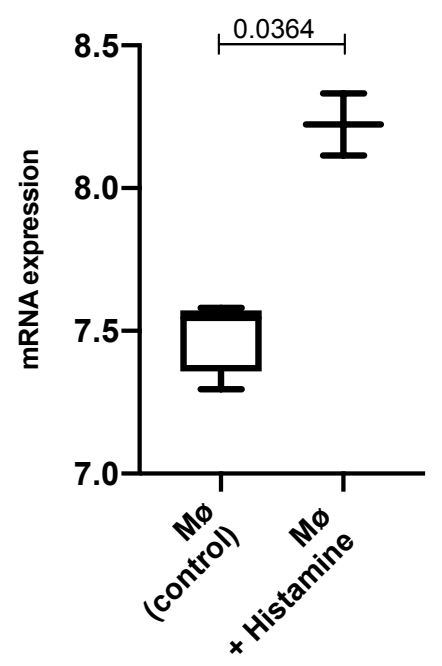

IL-12B

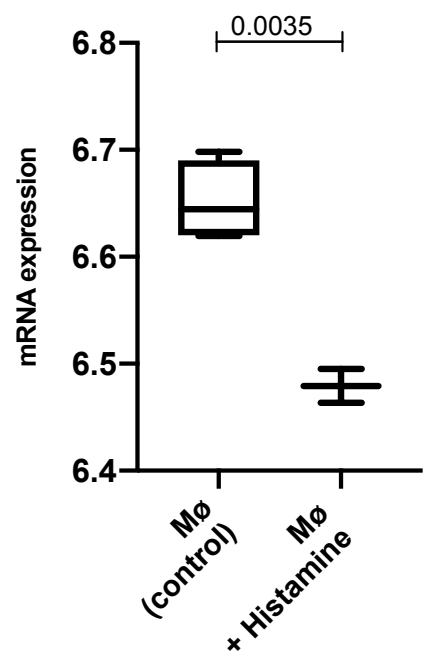

CXCL5

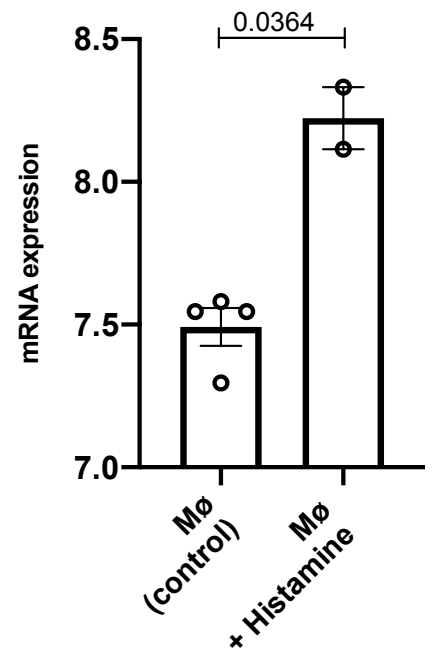

IL-12B

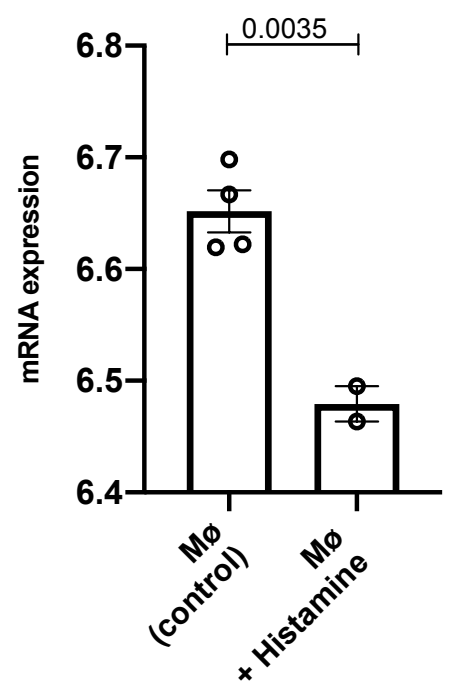

CXCL5

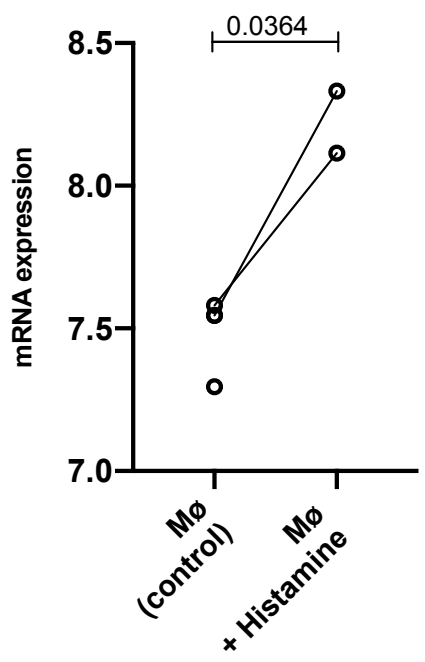

IL-12B

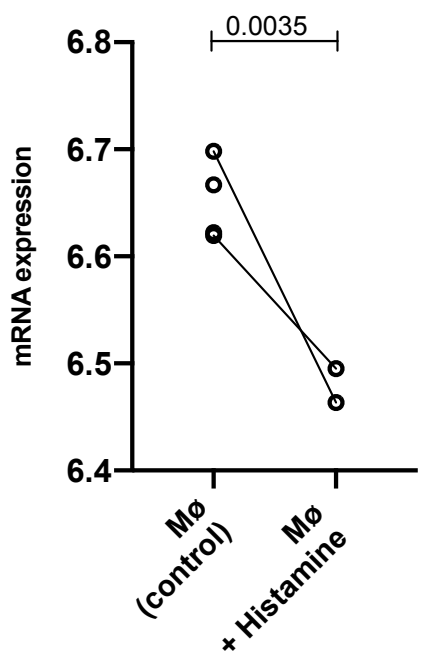

Figure 2: Immunity: Histamine induces the gene expression of CXCL5 while decreasing IL-12B expression.

The expression of the indicated genes is graphically represented by box and whisker plot from minimum to maximum value (left), by bar graph (middle) with the error bars denoting the standard error of the mean, and by symbols and connecting lines (right). For control samples, the two connecting samples on the right hand panel represent macrophages from Donor $A$ and Donor B with and without histamine treatment. There are two additional control samples, unconnected, representing mock treatment and mock histamine treatment. A statistical test was performed to determine if the difference in mRNA expression values between control and histamine-treated samples was significant; the result of this t-test is shown on each graph as a p-value. 
C1QBP

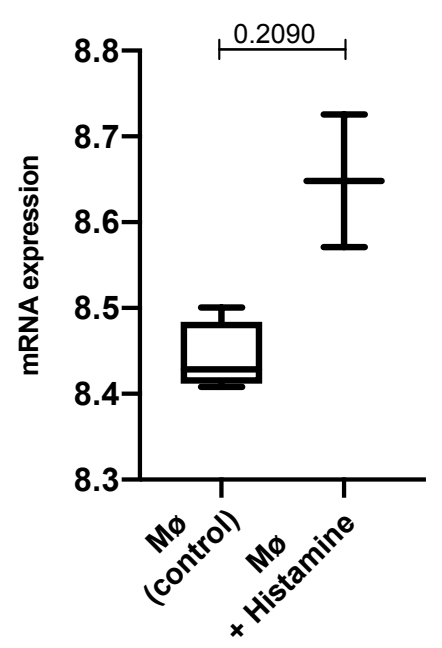

C1QTNF3

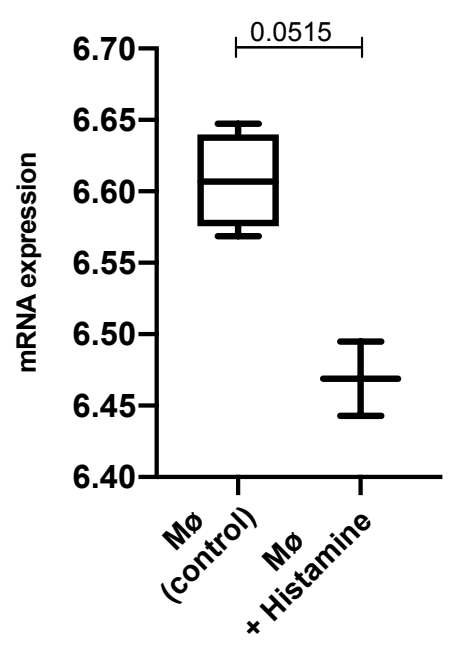

C1QBP

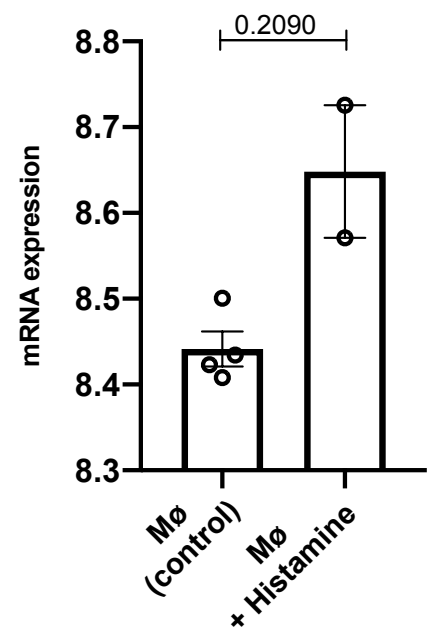

C1QTNF3

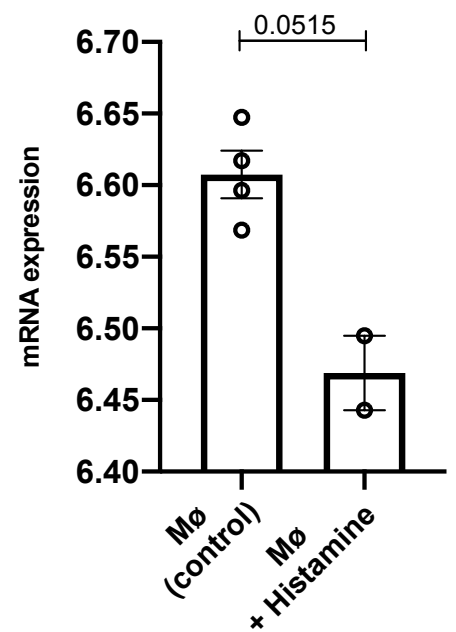

C1QBP

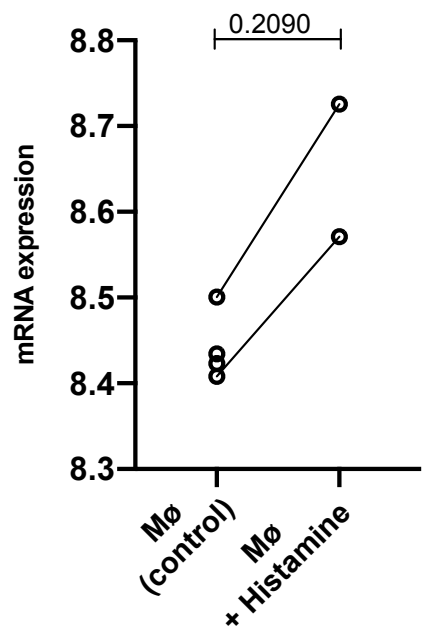

C1QTNF3

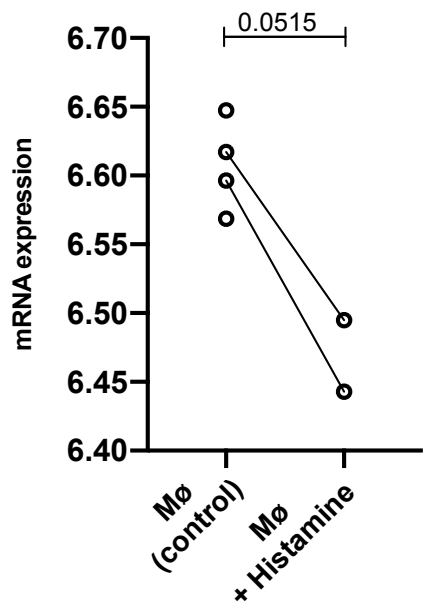

Figure 3: Immunity: Gene expression of complement cascade molecule C1QBP is activated while C1QTNF3 is repressed following histamine treatment.

The expression of the indicated genes is graphically represented by box and whisker plot from minimum to maximum value (left), by bar graph (middle) with the error bars denoting the standard error of the mean, and by symbols and connecting lines (right). For control samples, the two connecting samples on the right hand panel represent macrophages from Donor $A$ and Donor B with and without histamine treatment. There are two additional control samples, unconnected, representing mock treatment and mock histamine treatment. A statistical test was performed to determine if the difference in mRNA expression values between control and histamine-treated samples was significant; the result of this t-test is shown on each graph as a p-value. 
CTSL

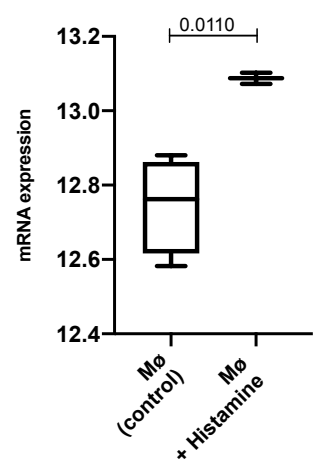

DEFB4A

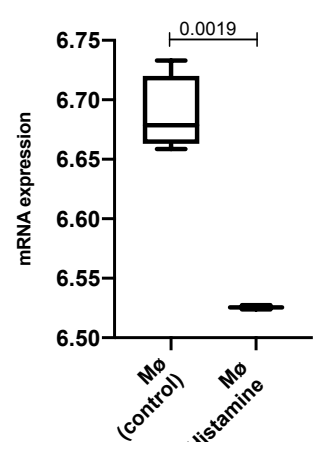

TRIL

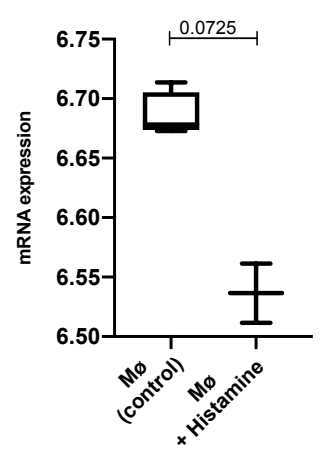

CTSL

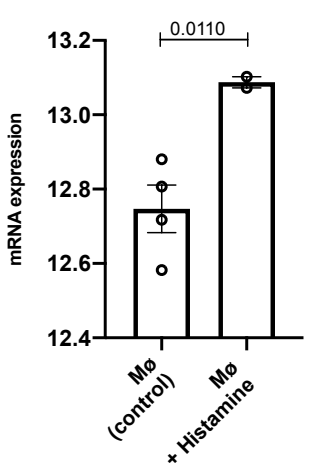

DEFB4A

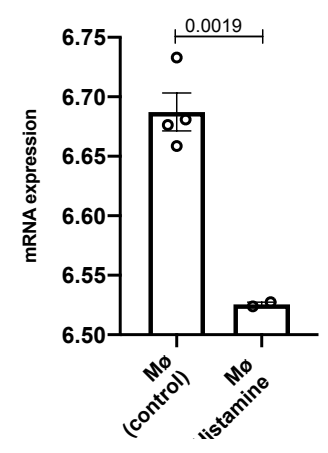

TRIL

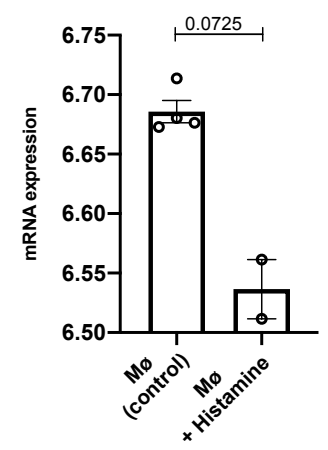

CTSL
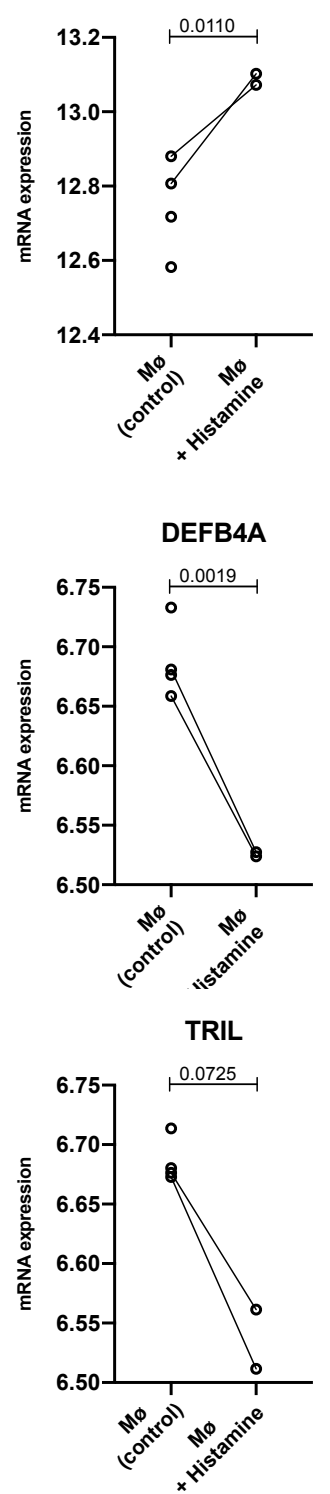

Figure 4: Immunity: Cathepsin $L$ is transcriptionally activated but defensin DEFB4A and innate immune effector TRIL is repressed following histamine exposure in macrophages.

The expression of the indicated genes is graphically represented by box and whisker plot from minimum to maximum value (left), by bar graph (middle) with the error bars denoting the standard error of the mean, and by symbols and connecting lines (right). For control samples, the two connecting samples on the right hand panel represent macrophages from Donor $A$ and Donor B with and without histamine treatment. There are two additional control samples, unconnected, representing mock treatment and mock histamine treatment. A statistical test was performed to determine if the difference in mRNA expression values between control and histamine-treated samples was significant; the result of this t-test is shown on each graph as a $\mathrm{p}$-value. 

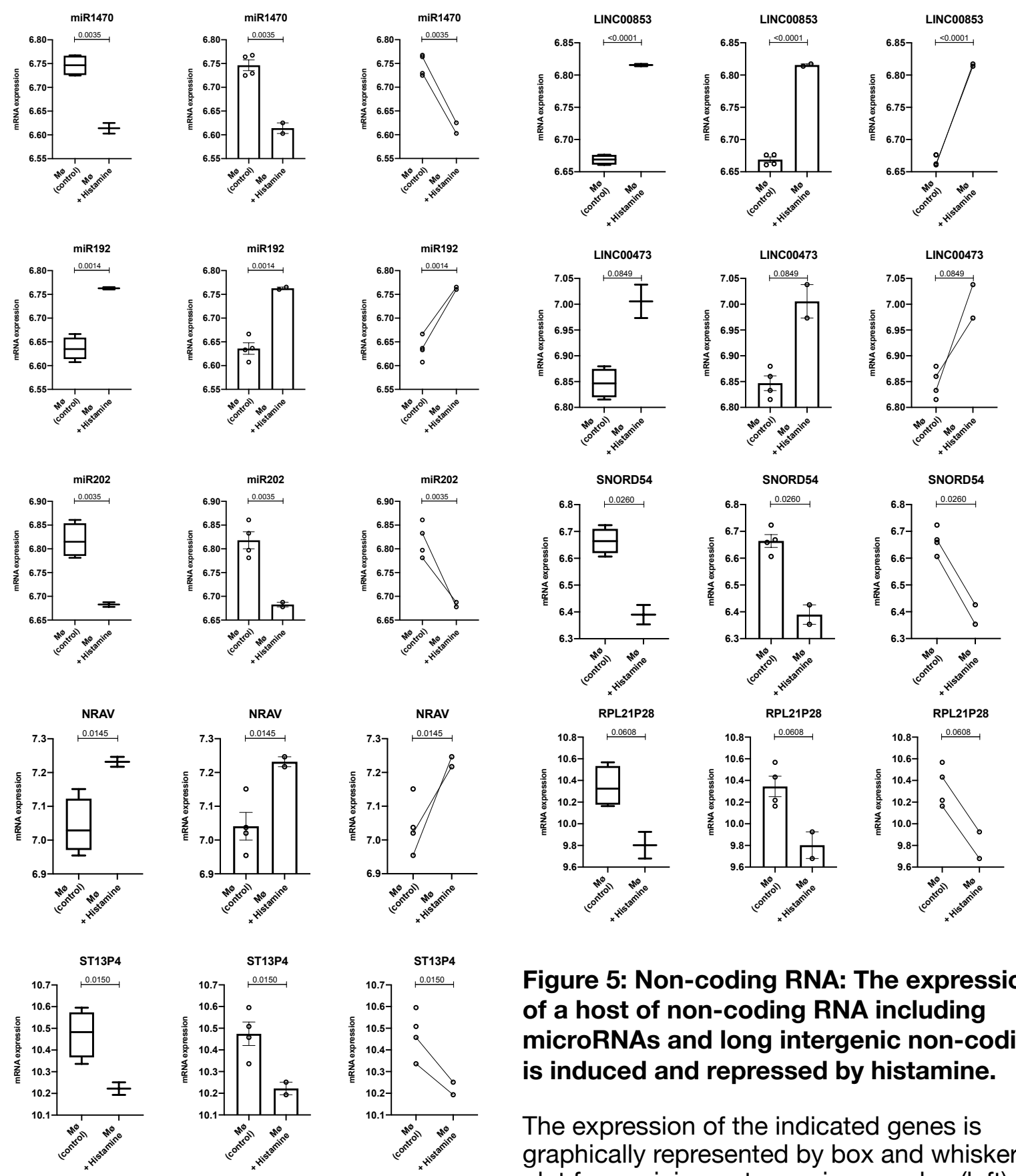

Figure 5: Non-coding RNA: The expression of a host of non-coding RNA including microRNAs and long intergenic non-coding is induced and repressed by histamine.

The expression of the indicated genes is graphically represented by box and whisker plot from minimum to maximum value (left), by bar graph (middle) with the error bars denoting

the standard error of the mean, and by symbols and connecting lines (right). For control samples, the two connecting samples on the right hand panel represent macrophages from Donor A and Donor B with and without histamine treatment. There are two additional control samples, unconnected, representing mock treatment and mock histamine treatment. A statistical test was performed to determine if the difference in mRNA expression values between control and histamine-treated samples was significant; the result of this t-test is shown on each graph as a p-value. 

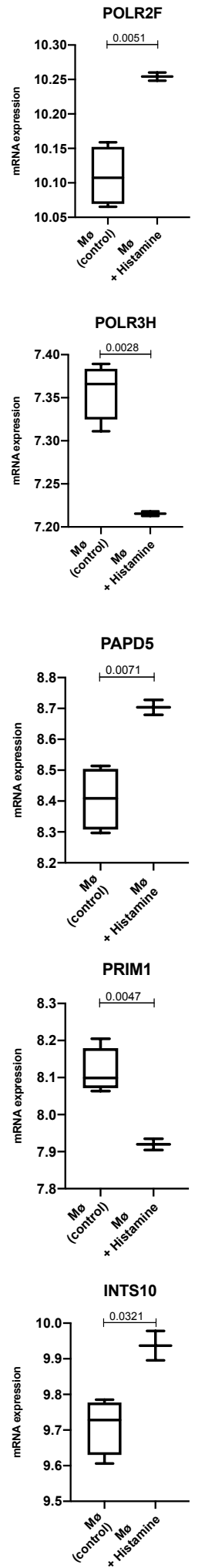
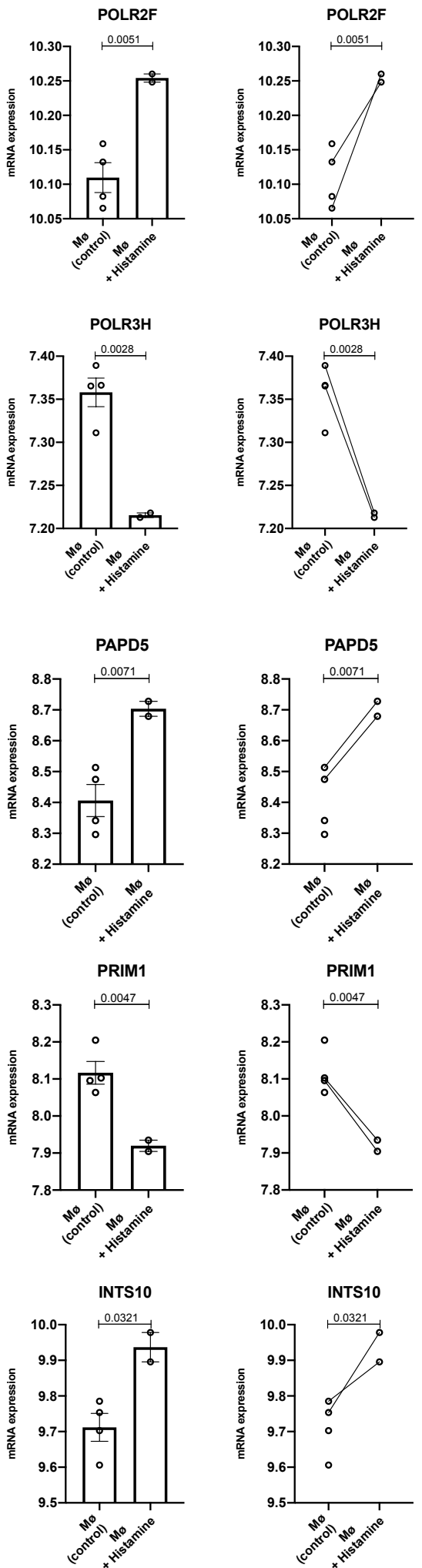

Figure 6: RNA Polymerases: Levels of RNA Polymerase POLR2F increase while levels of POLR3H decrease in macrophages, and other polymerase associated genes are differentially expressed in macrophages following histamine exposure.

The expression of the indicated genes is graphically represented by box and whisker plot from minimum to maximum value (left), by bar graph (middle) with the error bars denoting the standard error of the mean, and by symbols and connecting lines (right). For control samples, the two connecting samples on the right hand panel represent macrophages from Donor A and Donor B with and without histamine treatment.

There are two additional control samples, unconnected, representing mock treatment and mock histamine treatment. A statistical test was performed to determine if the difference in mRNA expression values between control and histamine-treated samples was significant; the result of this t-test is shown on each graph as a $p$-value. 

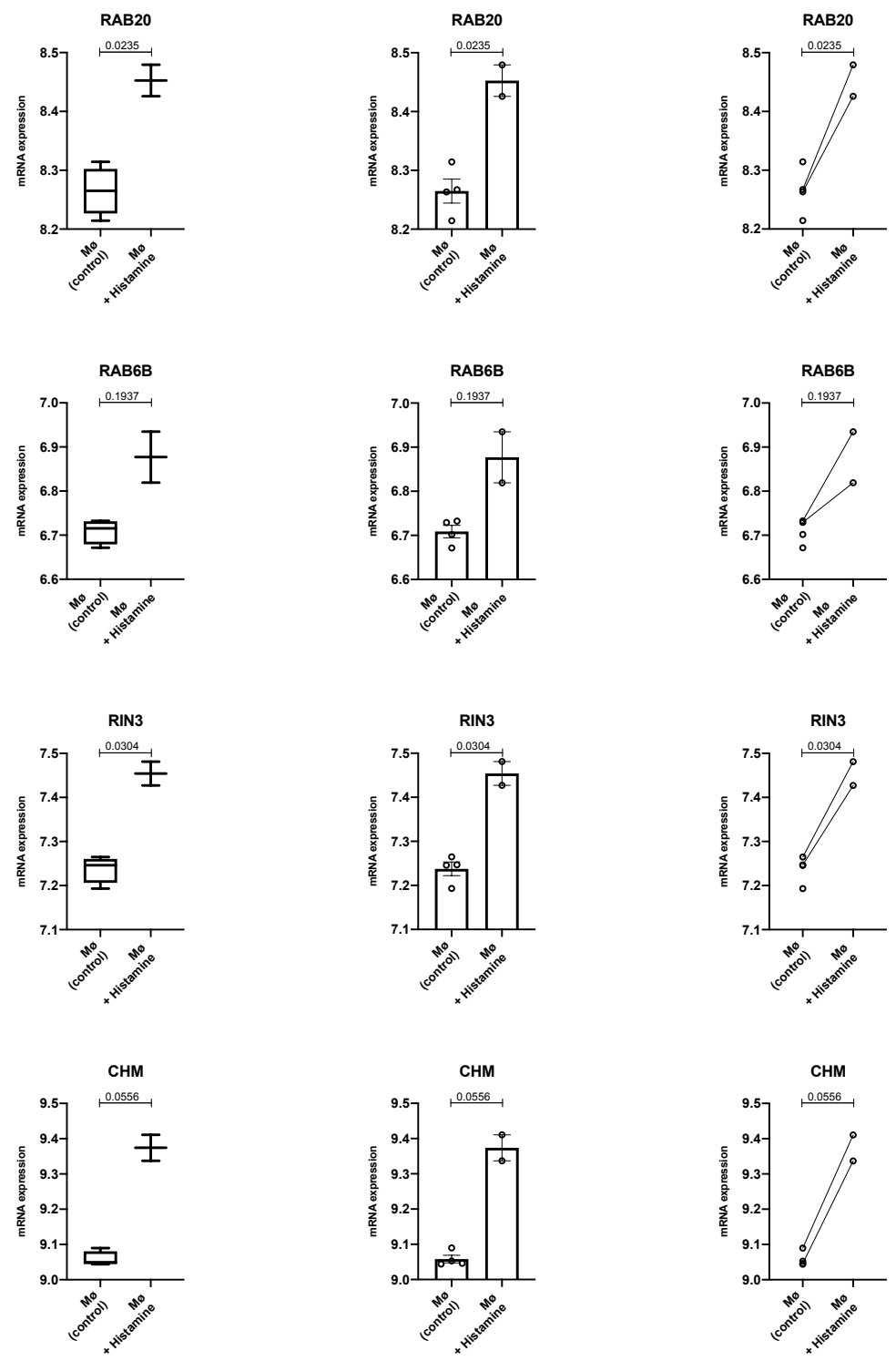

Figure 7: Rab family genes: Histamine activates gene expression of RAB6B and RAB20 as well as two effectors in the RAB family

The expression of the indicated genes is graphically represented by box and whisker plot from minimum to maximum value (left), by bar graph (middle) with the error bars denoting the standard error of the mean, and by symbols and connecting lines (right). For control samples, the two connecting samples on the right hand panel represent macrophages from Donor $A$ and Donor B with and without histamine treatment. There are two additional control samples, unconnected, representing mock treatment and mock histamine treatment. A statistical test was performed to determine if the difference in mRNA expression values between control and histamine-treated samples was significant; the result of this t-test is shown on each graph as a p-value. 

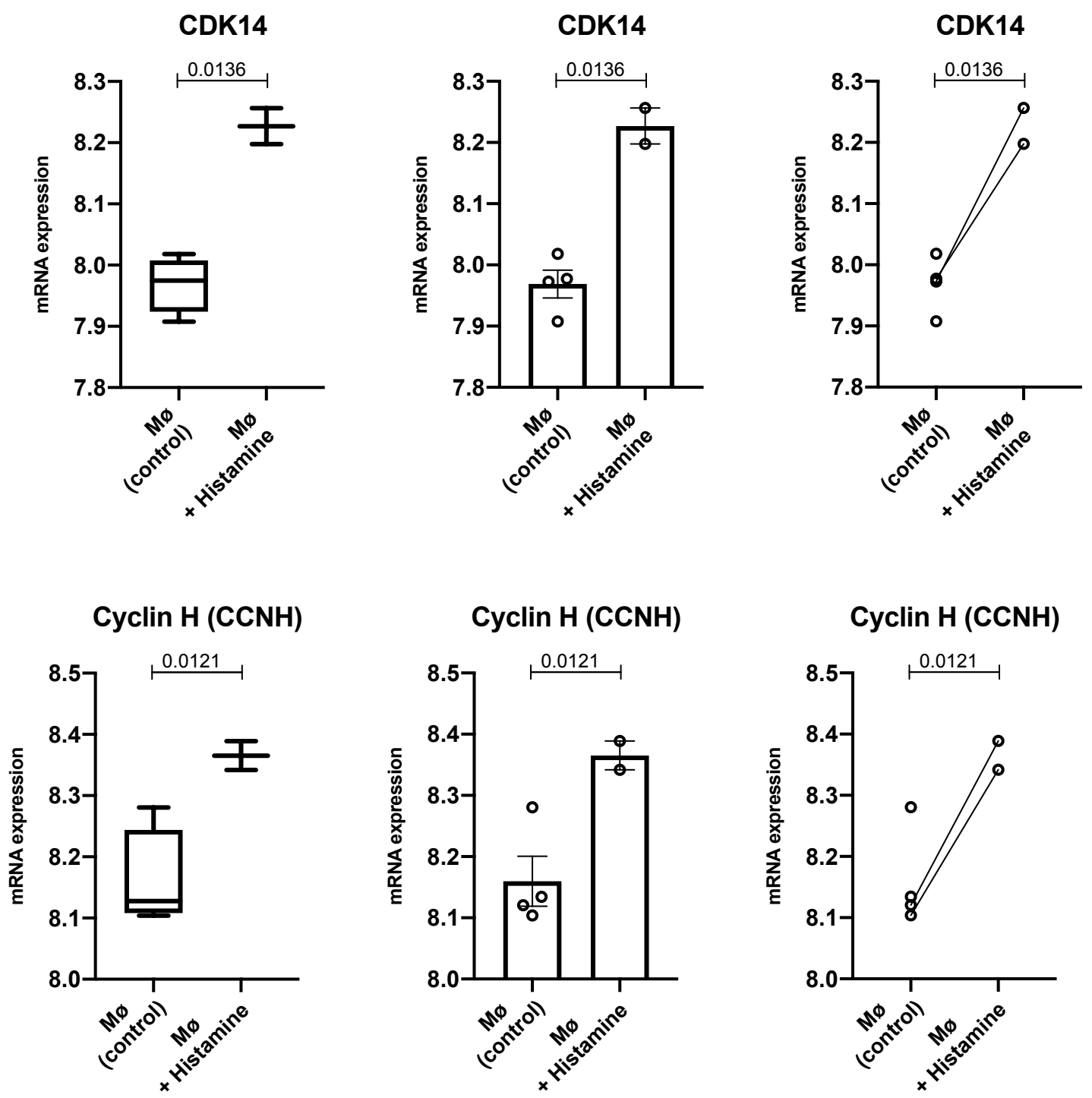

Figure 8: The Cell Cycle: CDK14 and Cyclin $\mathrm{H}$ are transcriptionally activated following histamine exposure in macrophages.

The expression of the indicated genes is graphically represented by box and whisker plot from minimum to maximum value (left), by bar graph (middle) with the error bars denoting the standard error of the mean, and by symbols and connecting lines (right). For control samples, the two connecting samples on the right hand panel represent macrophages from Donor A and Donor B with and without histamine treatment. There are two additional control samples, unconnected, representing mock treatment and mock histamine treatment. A statistical test was performed to determine if the difference in mRNA expression values between control and histamine-treated samples was significant; the result of this t-test is shown on each graph as a p-value. 

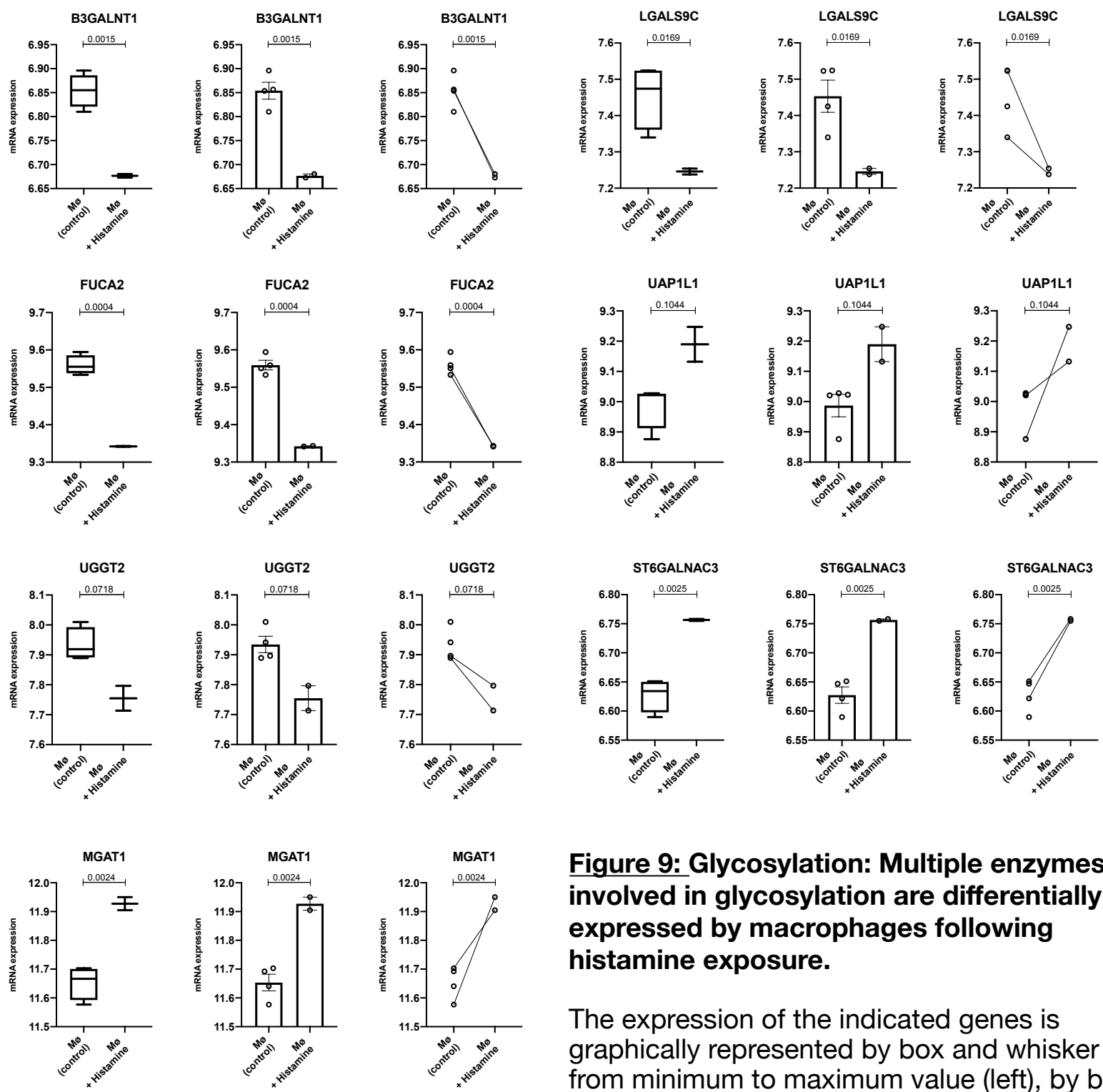

Figure 9: Glycosylation: Multiple enzymes involved in glycosylation are differentially expressed by macrophages following histamine exposure.

The expression of the indicated genes is graphically represented by box and whisker plot from minimum to maximum value (left), by bar graph (middle) with the error bars denoting the
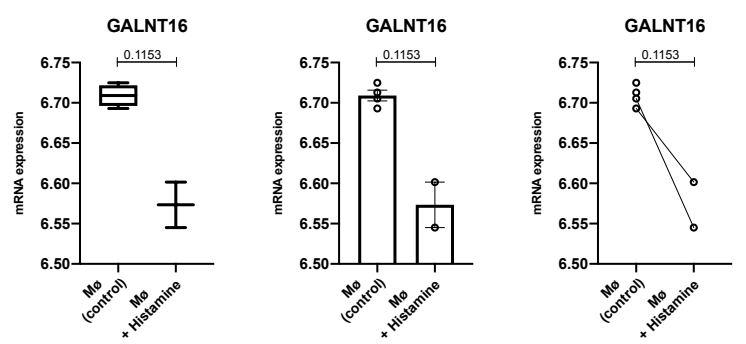
standard error of the mean, and by symbols and connecting lines (right). For control samples, the two connecting samples on the right hand panel represent macrophages from Donor $A$ and Donor B with and without histamine treatment. There are two additional control samples, unconnected, representing mock treatment and mock histamine treatment. A statistical test was performed to determine if the difference in mRNA expression values between control and histamine-treated samples was significant; the result of this t-test is shown on each graph as a $p$-value. 

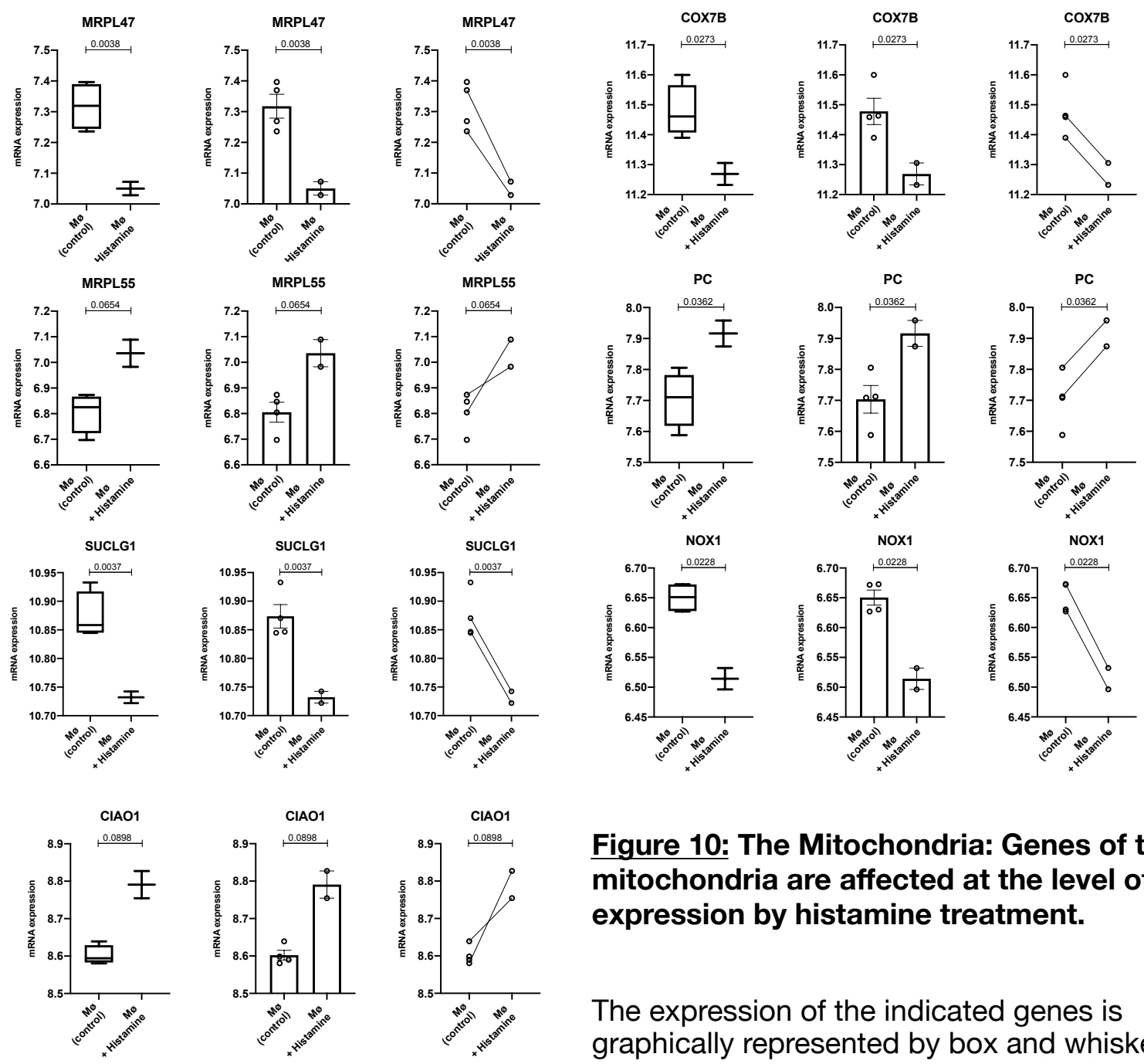

Figure 10: The Mitochondria: Genes of the mitochondria are affected at the level of RNA expression by histamine treatment.
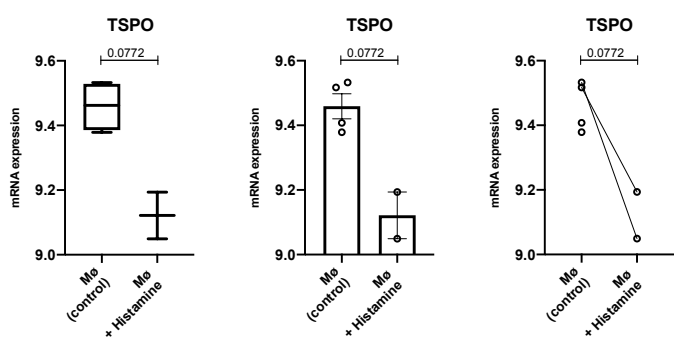

The expression of the indicated genes is graphically represented by box and whisker plot from minimum to maximum value (left), by bar graph (middle) with the error bars denoting the standard error of the mean, and by symbols and connecting lines (right). For control samples, the two connecting samples on the right hand panel represent macrophages from Donor $A$ and Donor $B$ with and without histamine treatment. There are two additional control samples, unconnected, representing mock treatment and mock histamine
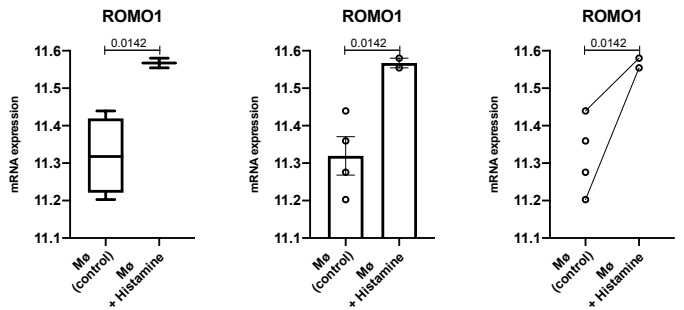

treatment. A statistical test was performed to determine if the difference in mRNA expression values between control and histamine-treated samples was significant; the result of this t-test is shown on each graph as a $p$-value. 

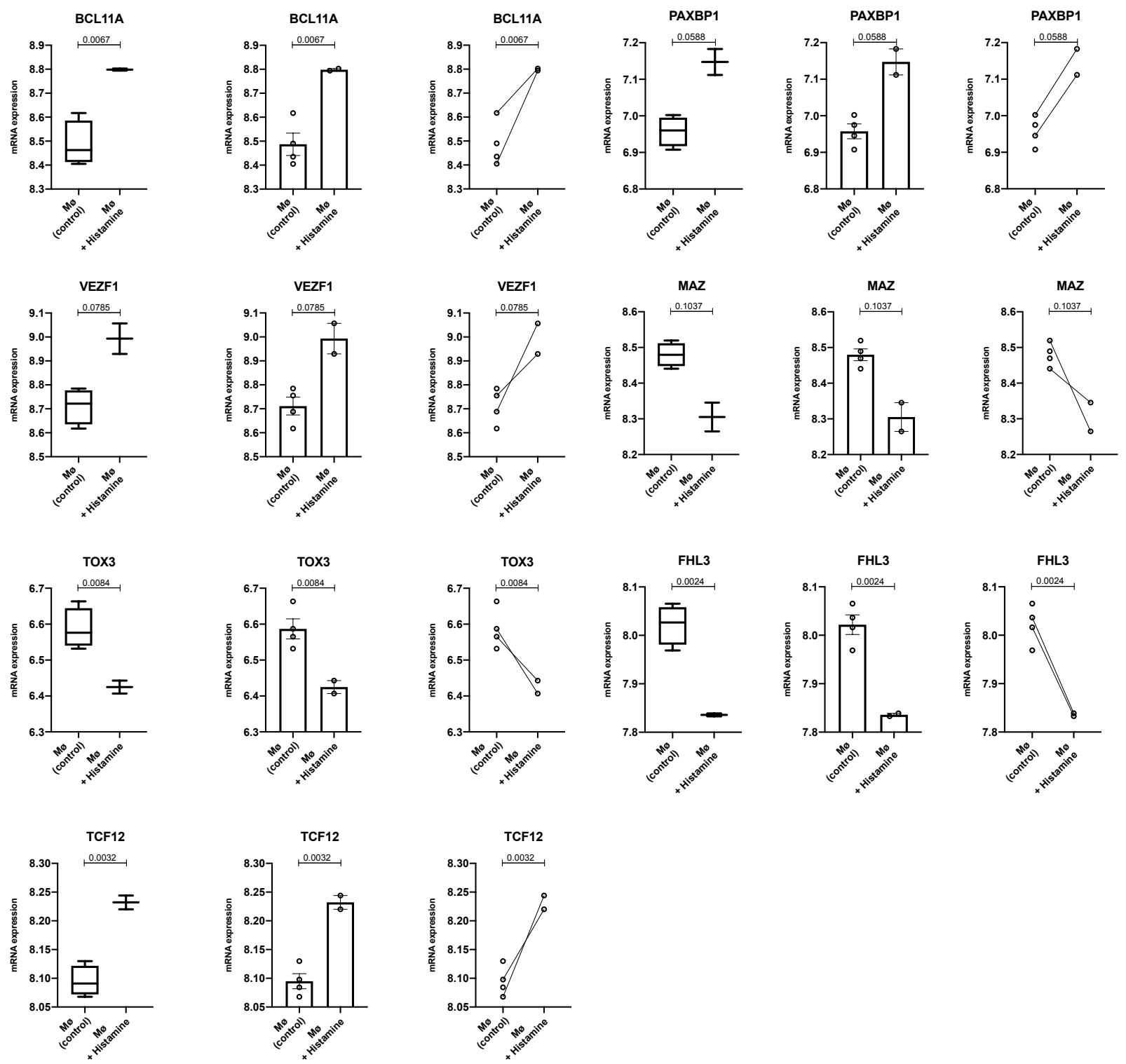

Figure 11: Transcription Factors: The expression of multiple transcription factors is activated by histamine in macrophages, but Tox 3 expression decreases

The expression of the indicated genes is graphically represented by box and whisker plot from minimum to maximum value (left), by bar graph (middle) with the error bars denoting the standard error of the mean, and by symbols and connecting lines (right). For control samples, the two connecting samples on the right hand panel represent macrophages from Donor $A$ and Donor B with and without histamine treatment. There are two additional control samples, unconnected, representing mock treatment and mock histamine treatment. A statistical test was performed to determine if the difference in mRNA expression values between control and histamine-treated samples was significant; the result of this t-test is shown on each graph as a p-value. 

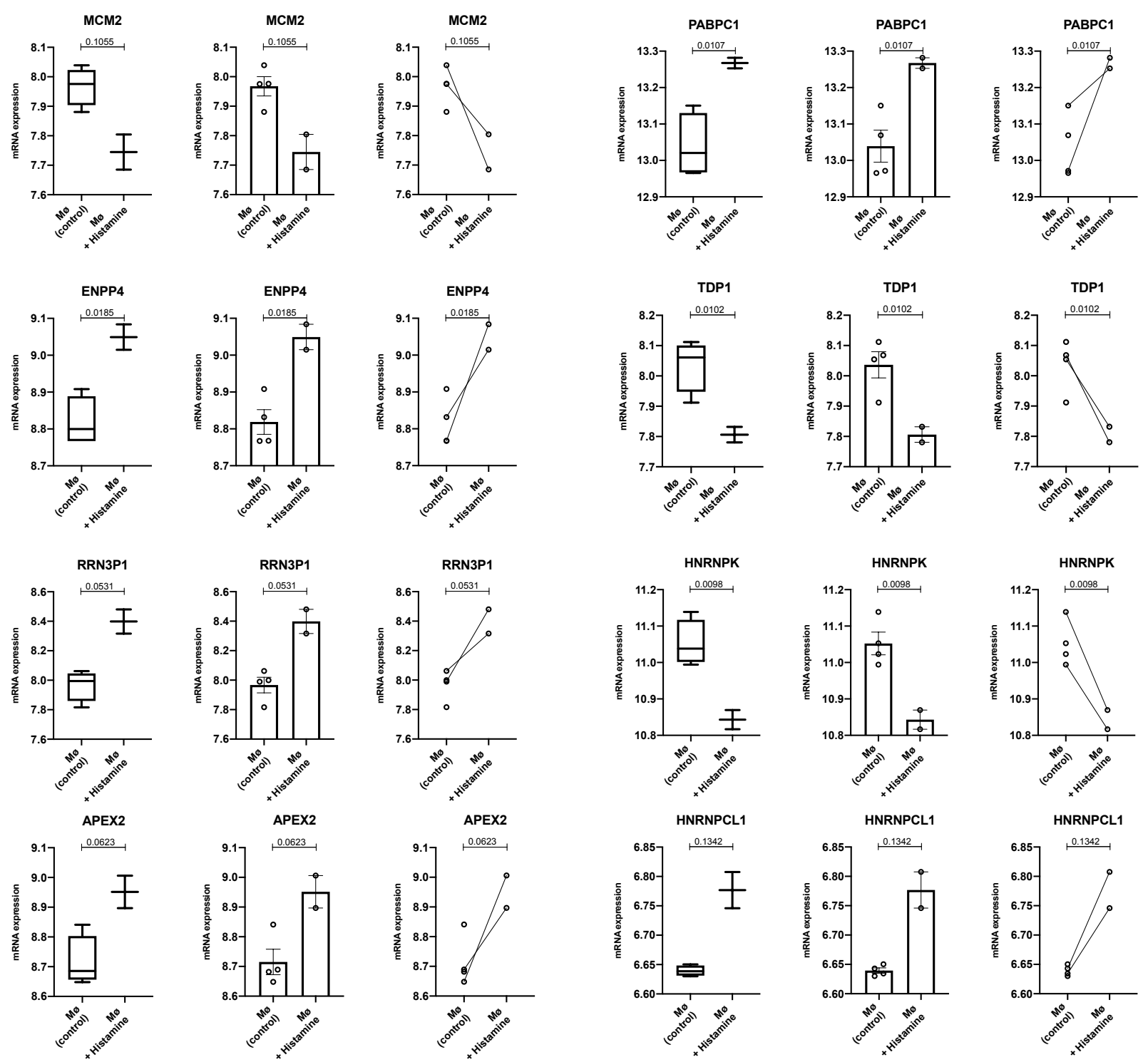

Figure 12: DNA and RNA: Molecules that replicate, repair, or bind DNA and RNA are
differentially expressed following histamine exposure in macrophages.

The expression of the indicated genes is graphically represented by box and whisker plot from minimum to maximum value (left), by bar graph (middle) with the error bars denoting the standard error of the mean, and by symbols and connecting lines (right). For control samples, the two connecting samples on the right hand panel represent macrophages from Donor $A$ and Donor B with and without histamine treatment. There are two additional control samples, unconnected, representing mock treatment and mock histamine treatment. A statistical test was performed to determine if the difference in mRNA expression values between control and histamine-treated samples was significant; the result of this t-test is shown on each graph as a p-value. 

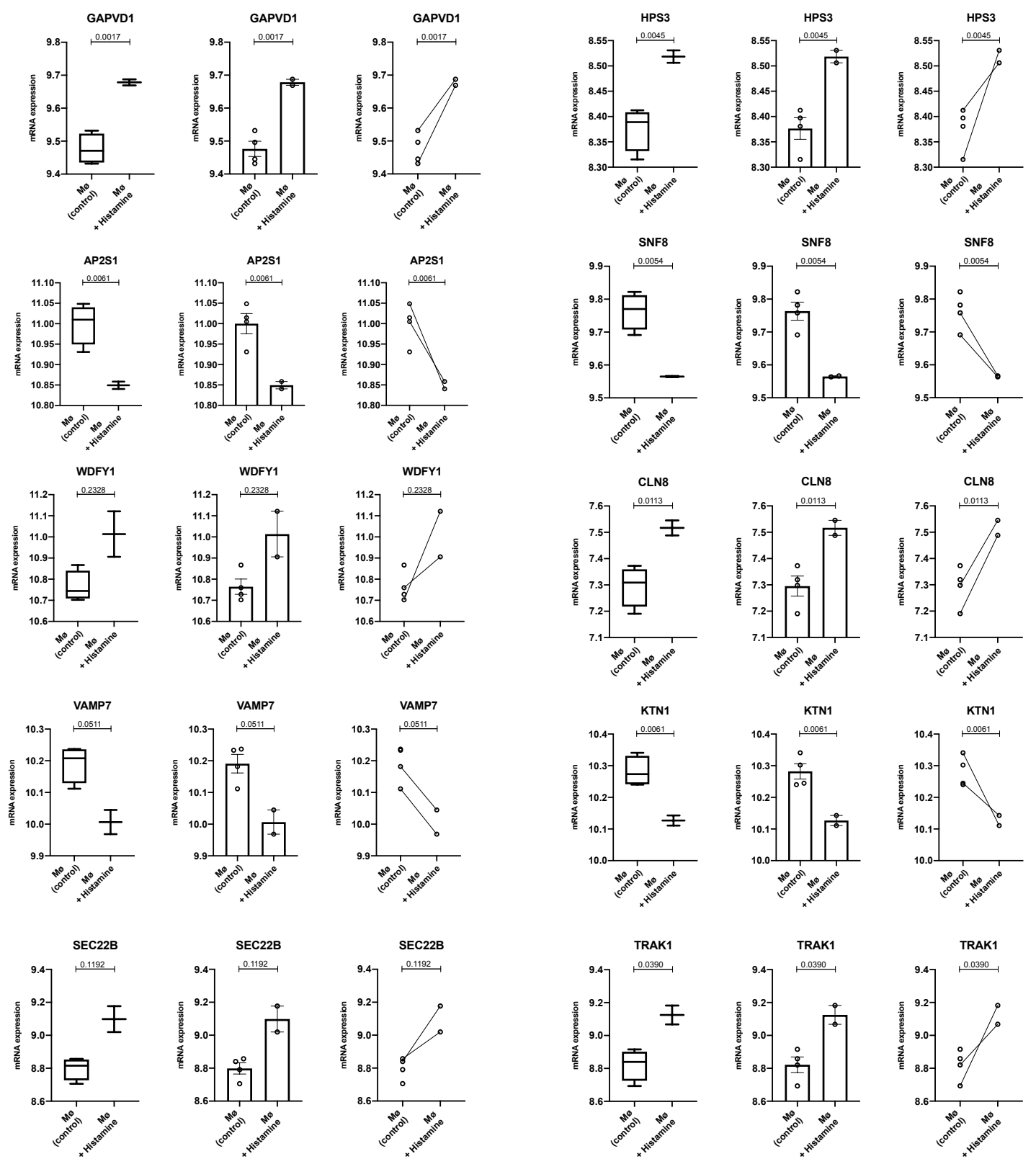

Figure 13: Cellular Transport: Cellular and vesicular trafficking gene expression is affected by histamine exposure

The expression of the indicated genes is graphically represented by box and whisker plot from minimum to maximum value (left), by bar graph (middle) with the error bars denoting the standard error of the mean, and by symbols and connecting lines (right). For control samples, the two connecting samples on the right hand panel represent macrophages from Donor $A$ and Donor B with and without histamine treatment. There are two additional control samples, unconnected, representing mock treatment and mock histamine treatment. A statistical test was performed to determine if the difference in mRNA expression values between control and histamine-treated samples was significant; the result of this t-test is shown on each graph as a p-value. 

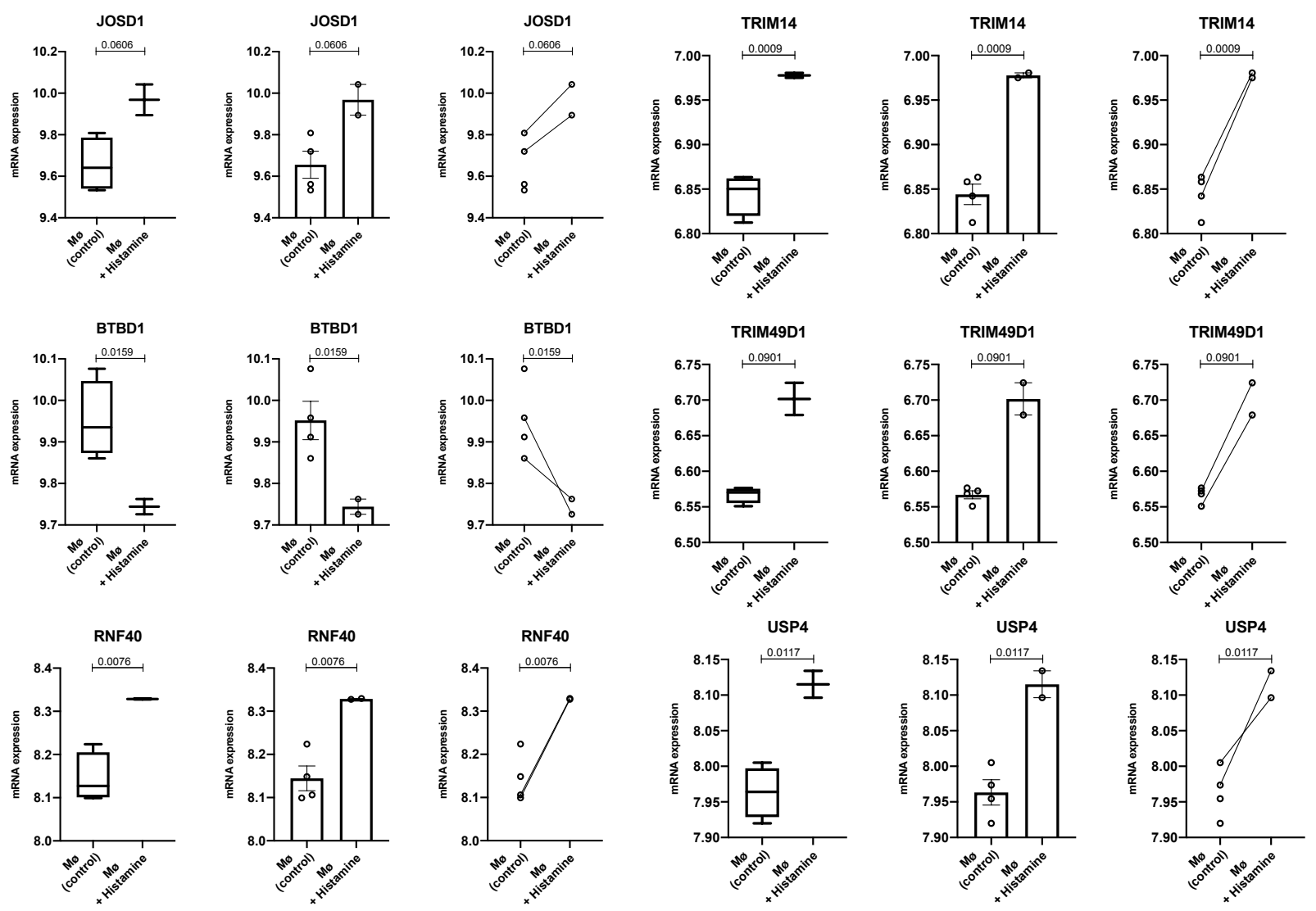

Figure 14: Ubiquitin: Multiple genes involved in ubiquitin modification are perturbed by histamine treatment in macrophages

The expression of the indicated genes is graphically represented by box and whisker plot from minimum to maximum value (left), by bar graph (middle) with the error bars denoting the standard error of the mean, and by symbols and connecting lines (right). For control samples, the two connecting samples on the right hand panel represent macrophages from Donor $A$ and Donor B with and without histamine treatment. There are two additional control samples, unconnected, representing mock treatment and mock histamine treatment. A statistical test was performed to determine if the difference in mRNA expression values between control and histamine-treated samples was significant; the result of this t-test is shown on each graph as a p-value. 

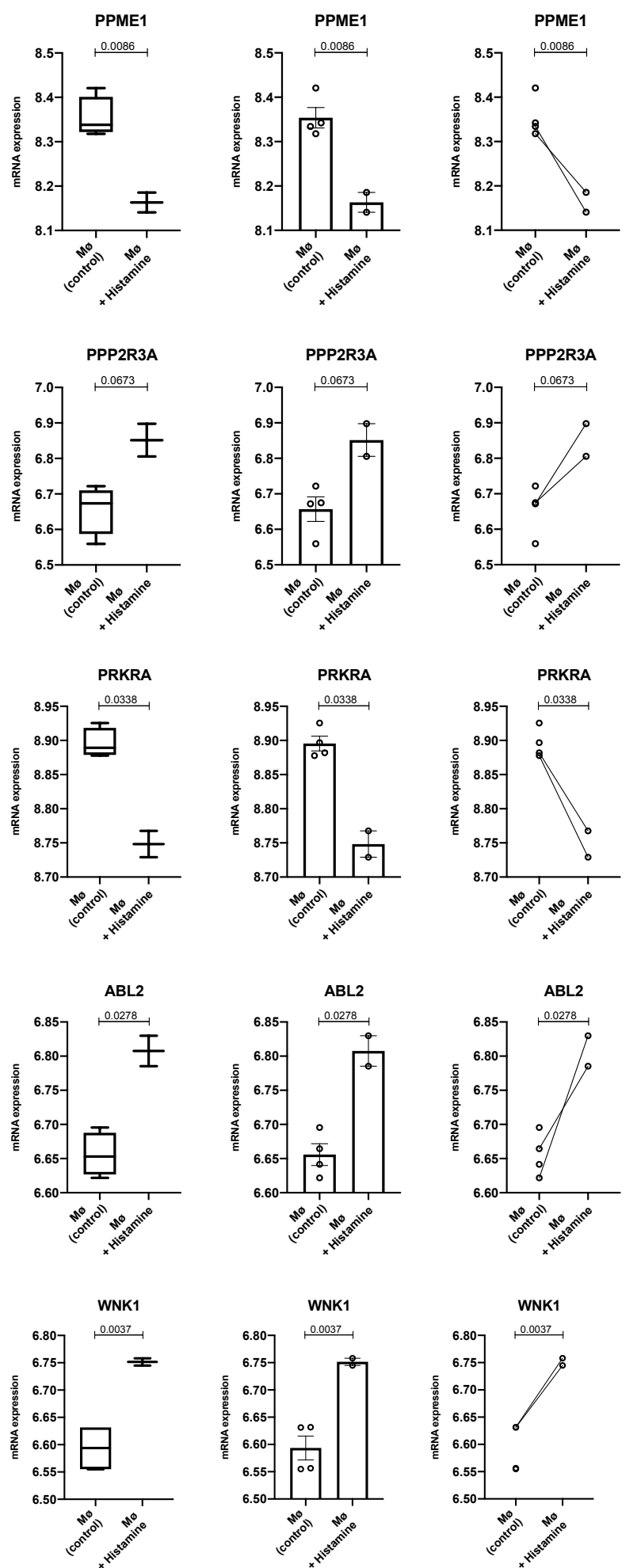

Figure 15: Phosphatases and Kinases: The gene expression of specific phosphatases and kinases is affected by histamine exposure

The expression of the indicated genes is graphically represented by box and whisker plot from minimum to maximum value (left), by bar graph (middle) with the error bars denoting the standard error of the mean, and by symbols and connecting lines (right). For control samples, the two connecting samples on the right hand panel represent macrophages from Donor A and Donor B with and without histamine treatment. There are two additional control samples, unconnected, representing mock treatment and mock histamine treatment. A statistical test was performed to determine if the difference in mRNA expression values between control and histamine-treated samples was significant; the result of this ttest is shown on each graph as a p-value. 

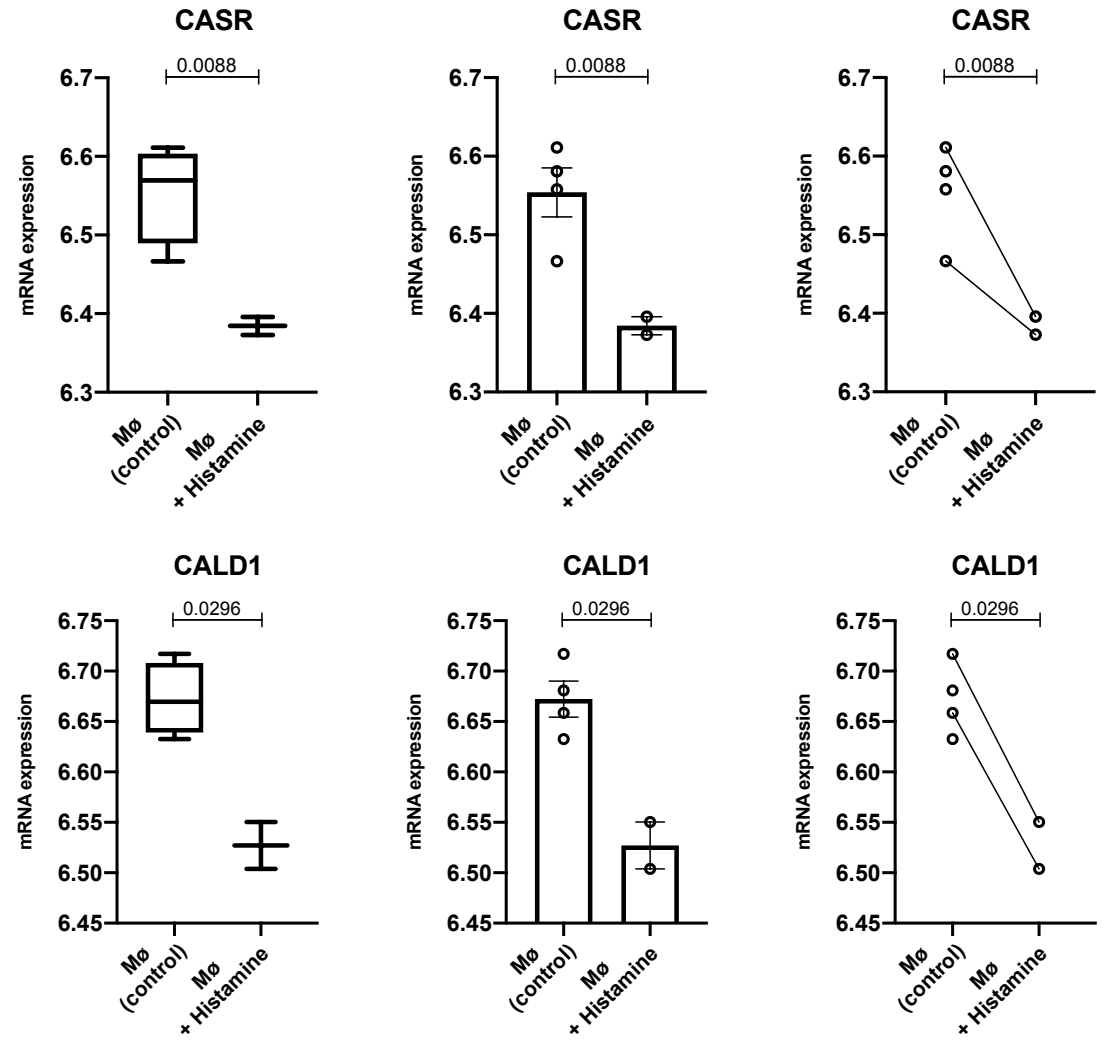

SRL

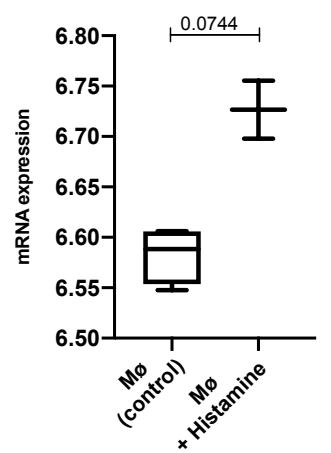

SRL
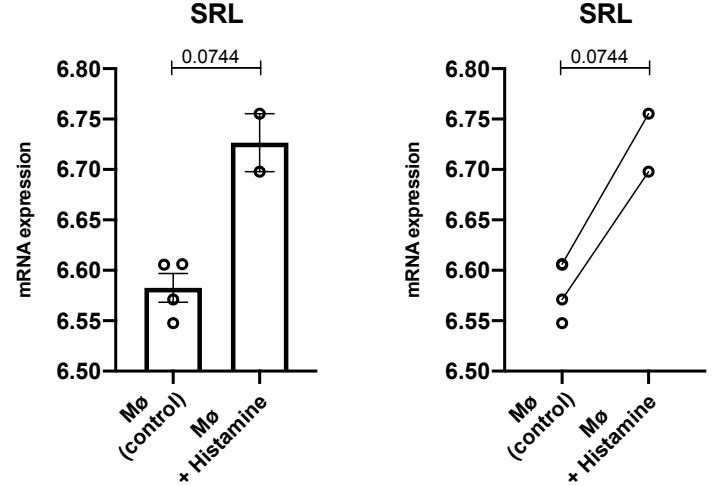

\section{Figure 16: Calcium signaling: Genes that modulate calcium signaling are affected by histamine exposure}

The expression of the indicated genes is graphically represented by box and whisker plot from minimum to maximum value (left), by bar graph (middle) with the error bars denoting the standard error of the mean, and by symbols and connecting lines (right). For control samples, the two connecting samples on the right hand panel represent macrophages from Donor $A$ and Donor B with and without histamine treatment. There are two additional control samples, unconnected, representing mock treatment and mock histamine treatment. A statistical test was performed to determine if the difference in mRNA expression values between control and histamine-treated samples was significant; the result of this t-test is shown on each graph as a p-value. 

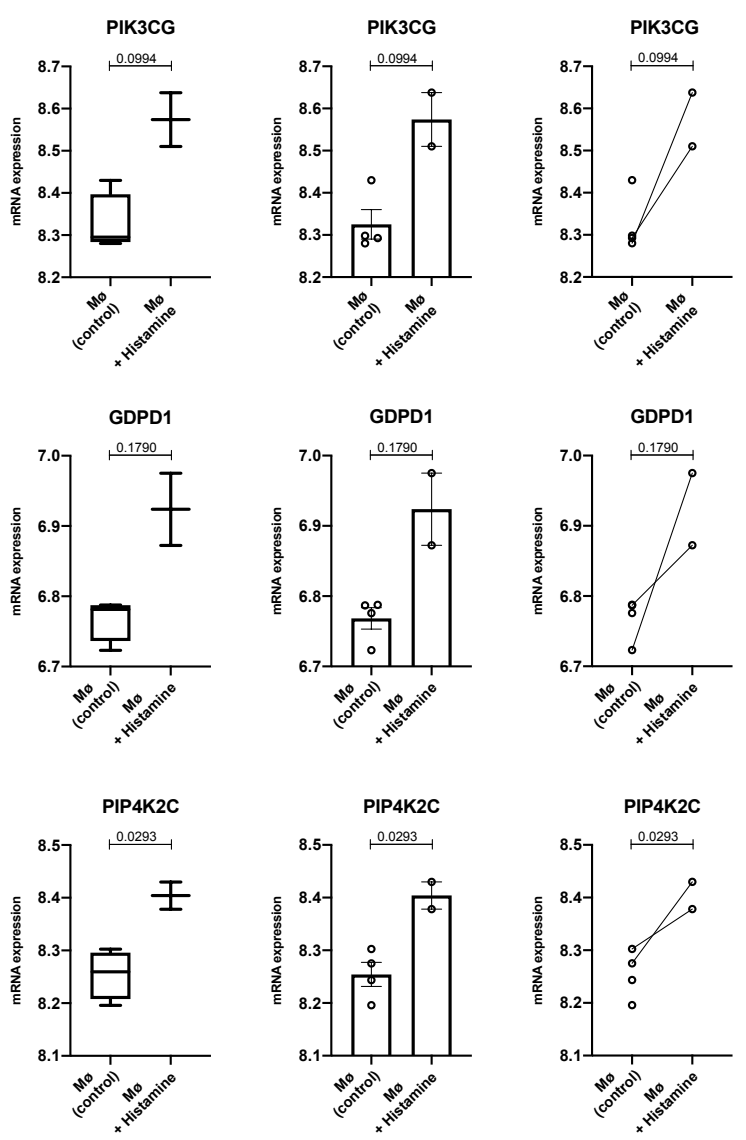

Figure 17: Phospholipids: Enzymes involved in manipulation of cellular phospholipids are affected at the level of gene expression by histamine

The expression of the indicated genes is graphically represented by box and whisker plot from minimum to maximum value (left), by bar graph (middle) with the error bars denoting the standard error of the mean, and by symbols and connecting lines (right). For control samples, the two connecting samples on the right hand panel represent macrophages from Donor $A$ and Donor B with and without histamine treatment. There are two additional control samples, unconnected, representing mock treatment and mock histamine treatment. A statistical test was performed to determine if the difference in mRNA expression values between control and histamine-treated samples was significant; the result of this t-test is shown on each graph as a p-value. 

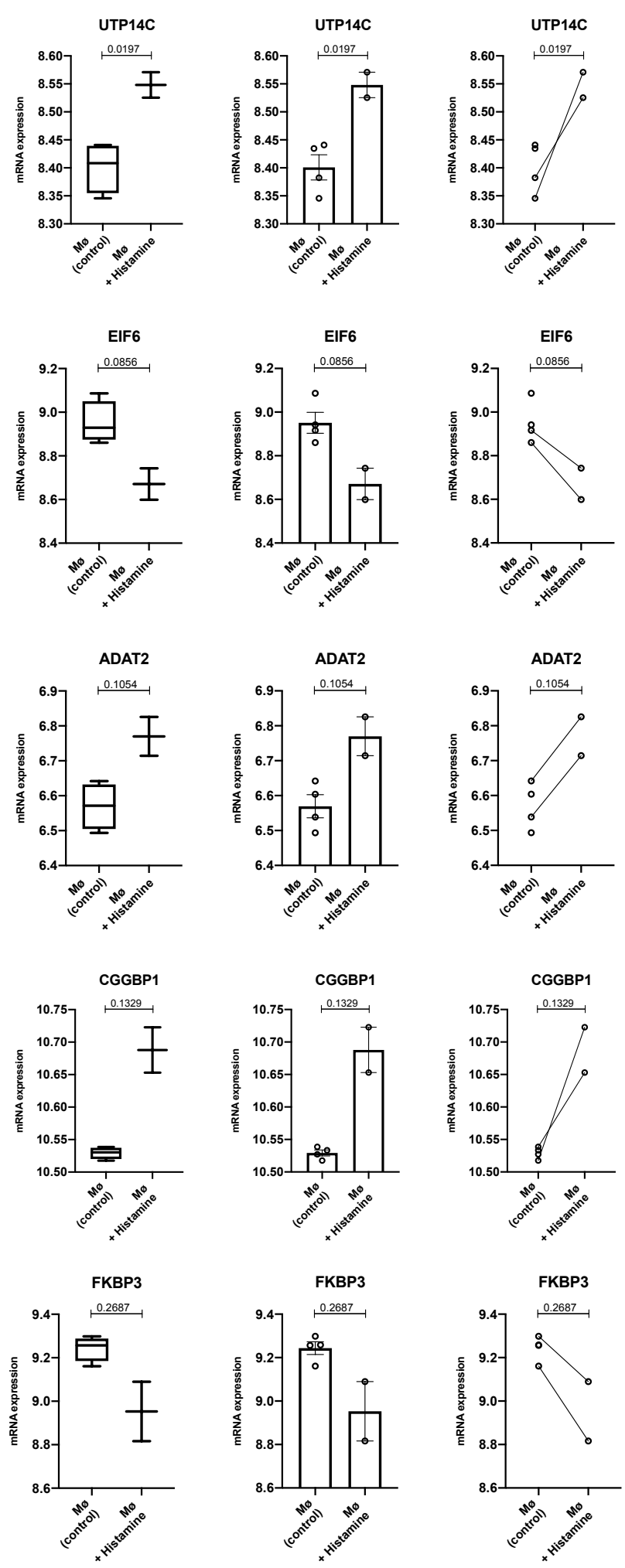

Figure 18: Translation and ER Stress: Histamine modulates that expression of genes involved in protein translation and quality control of proteins following cellular stress, including EDEM1 and EDEM3

The expression of the indicated genes is graphically represented by box and whisker plot from minimum to maximum value (left), by bar graph (middle) with the error bars denoting the standard error of the mean, and by symbols and connecting lines (right). For control samples, the two connecting samples on the right hand panel represent macrophages from Donor A and Donor B with and without histamine treatment. There are two additional control samples, unconnected, representing mock treatment and mock histamine treatment. A statistical test was performed to determine if the difference in mRNA expression values between control and histamine-treated samples was significant; the result of this ttest is shown on each graph as a p-value.
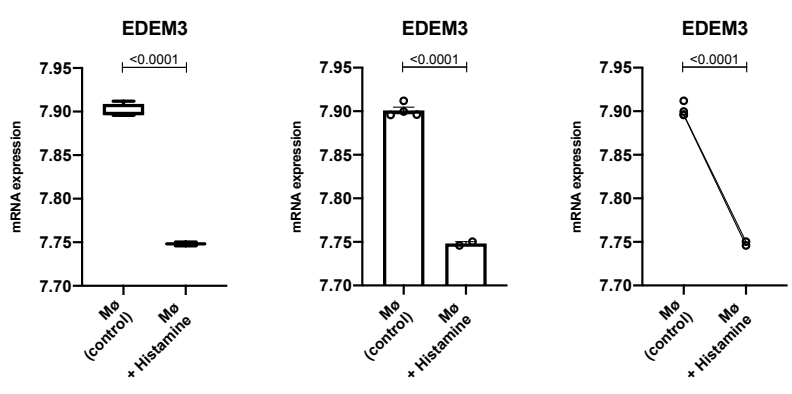
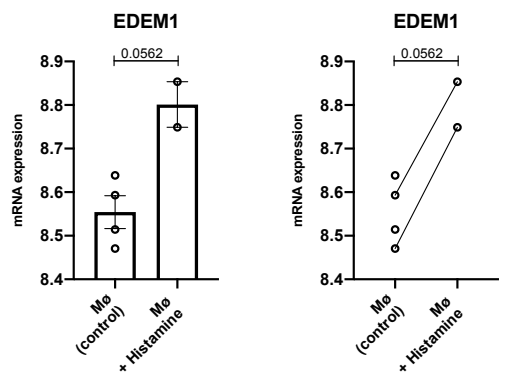

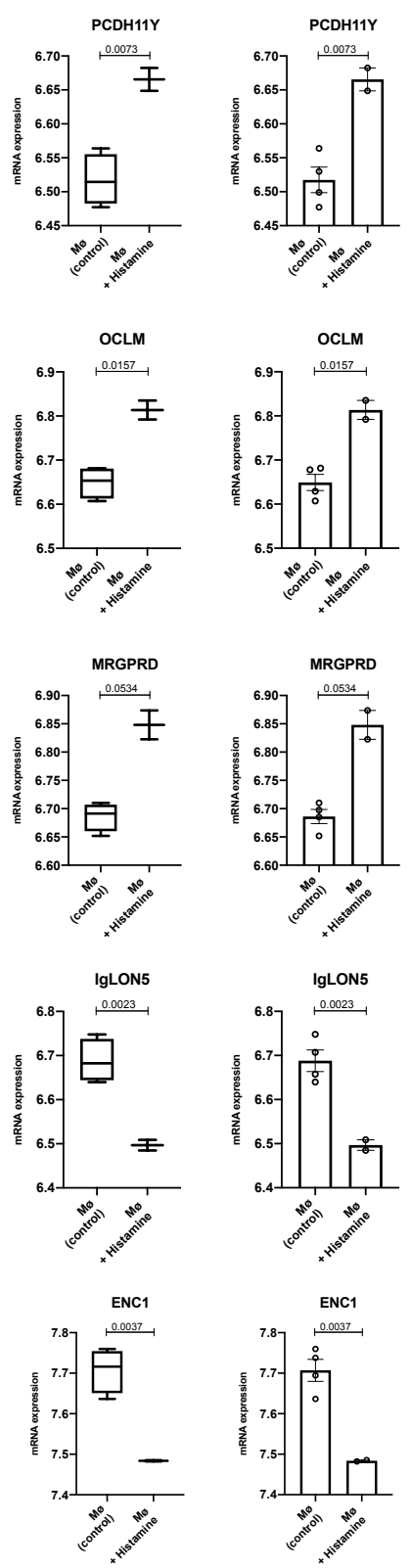
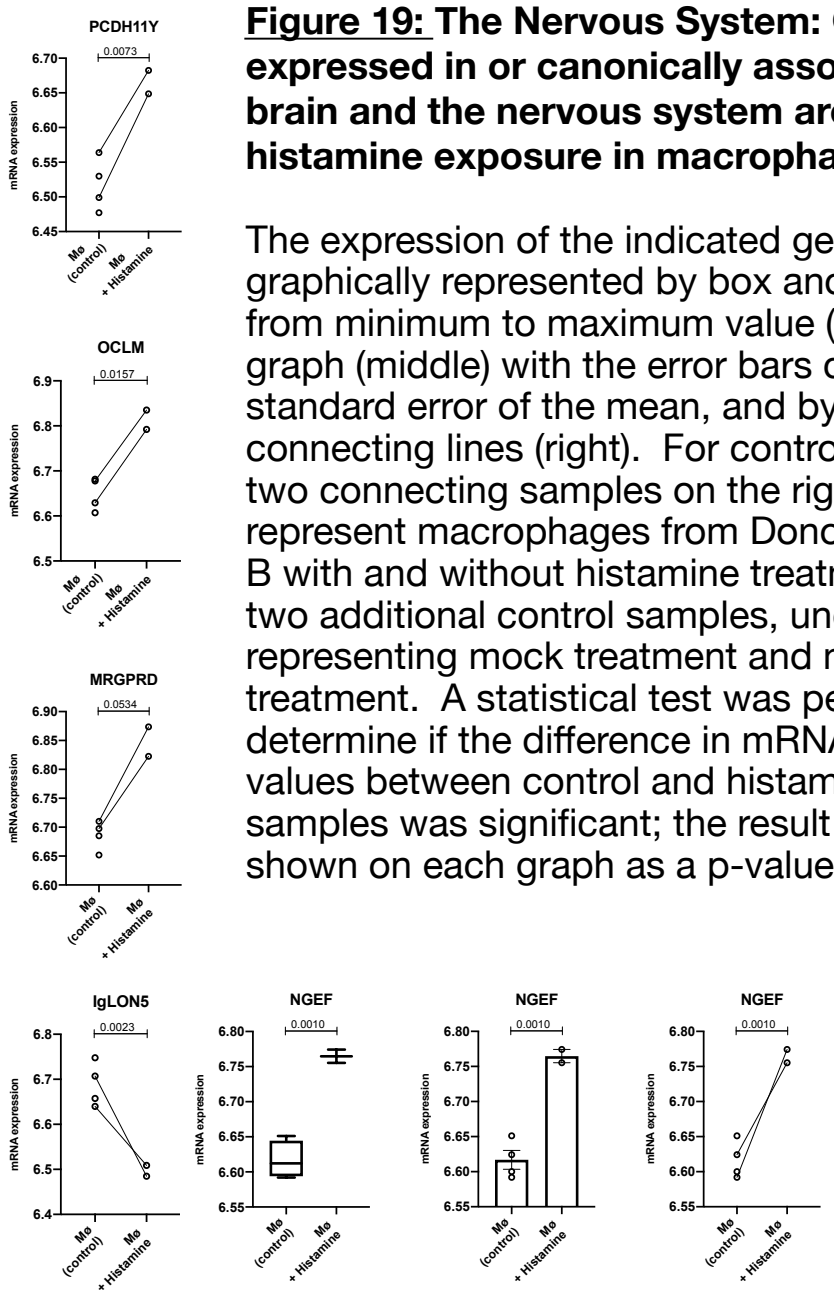

Figure 19: The Nervous System: Genes typically expressed in or canonically associated with the brain and the nervous system are affected by histamine exposure in macrophages

The expression of the indicated genes is graphically represented by box and whisker plot from minimum to maximum value (left), by bar graph (middle) with the error bars denoting the standard error of the mean, and by symbols and connecting lines (right). For control samples, the two connecting samples on the right hand panel represent macrophages from Donor A and Donor $B$ with and without histamine treatment. There are two additional control samples, unconnected, representing mock treatment and mock histamine treatment. A statistical test was performed to determine if the difference in mRNA expression values between control and histamine-treated samples was significant; the result of this t-test is shown on each graph as a p-value.

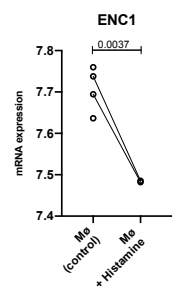

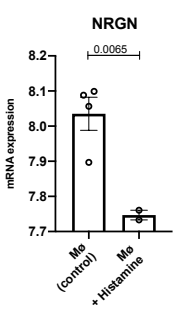



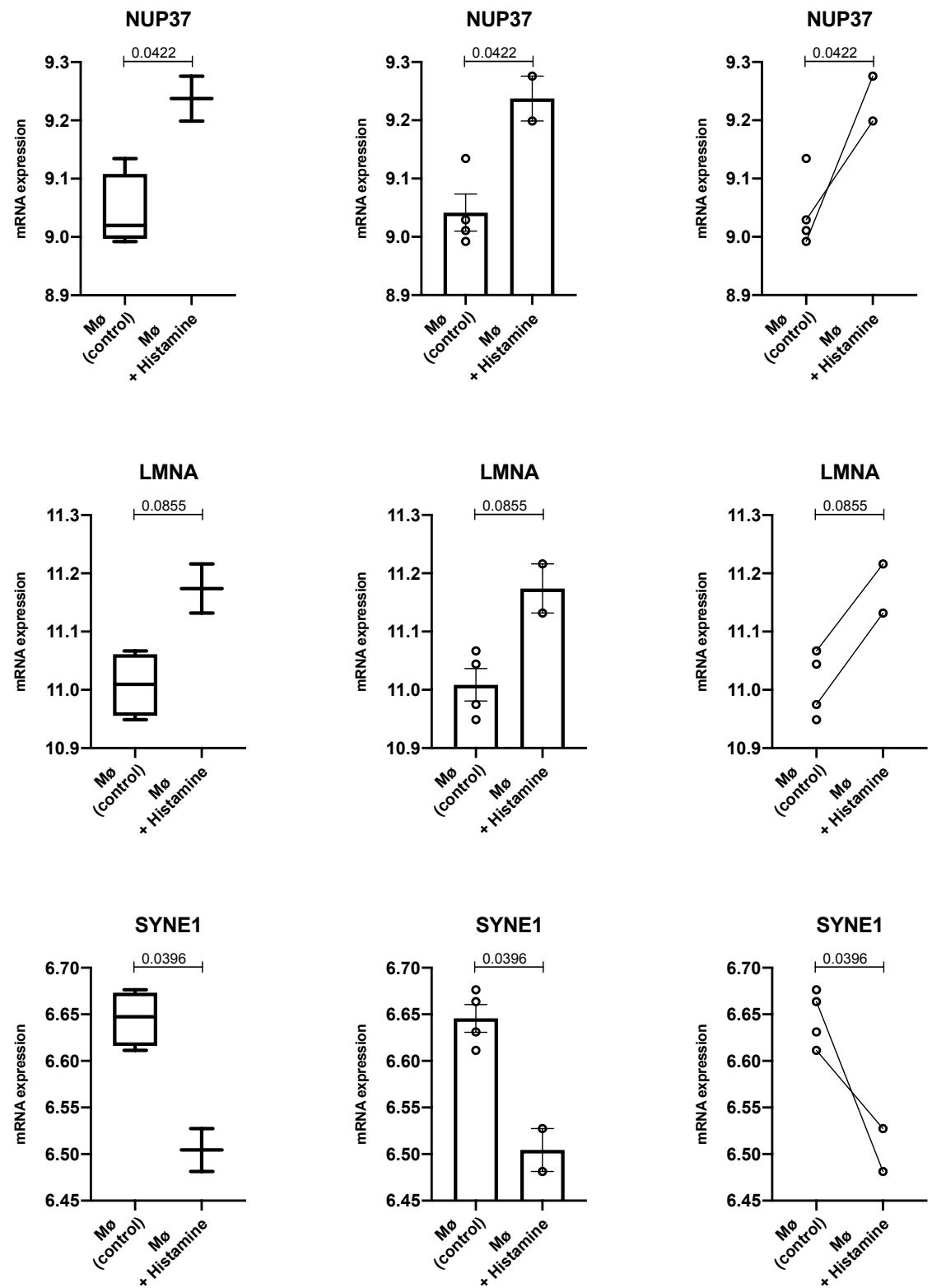

\section{Figure 20: The Nuclear Envelope: Nup37 and other genes at the nuclear envelope are affected by histamine exposure}

The expression of the indicated genes is graphically represented by box and whisker plot from minimum to maximum value (left), by bar graph (middle) with the error bars denoting the standard error of the mean, and by symbols and connecting lines (right). For control samples, the two connecting samples on the right hand panel represent macrophages from Donor $A$ and Donor B with and without histamine treatment. There are two additional control samples, unconnected, representing mock treatment and mock histamine treatment. A statistical test was performed to determine if the difference in mRNA expression values between control and histamine-treated samples was significant; the result of this t-test is shown on each graph as a p-value. 
TTC17

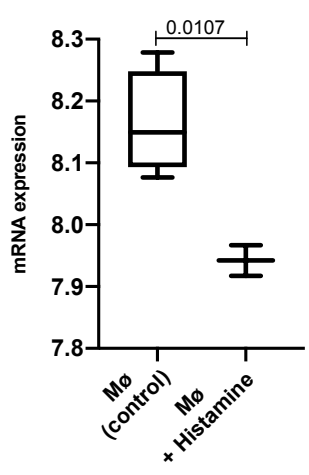

SPC25

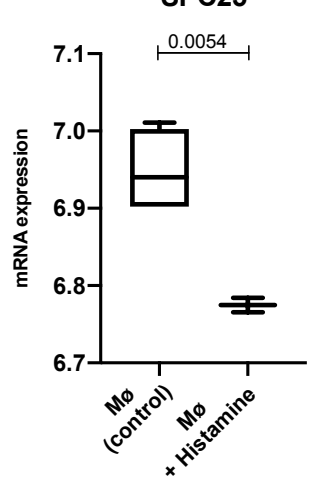

SRFBP1

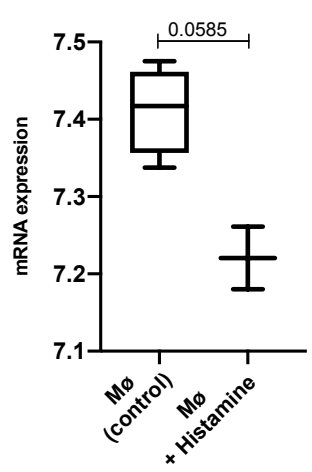

TTC17

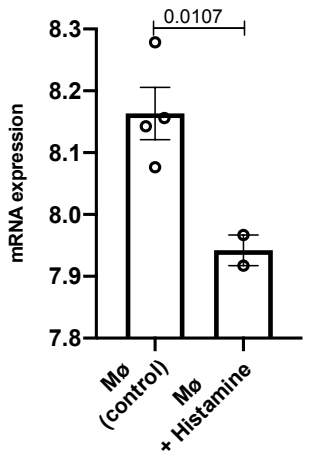

SPC25

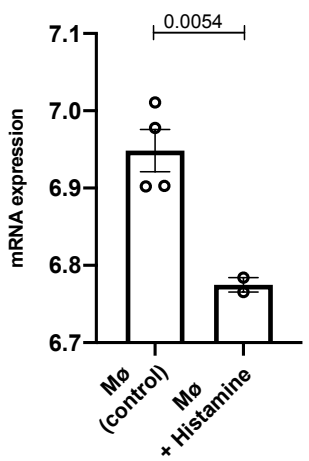

SRFBP1

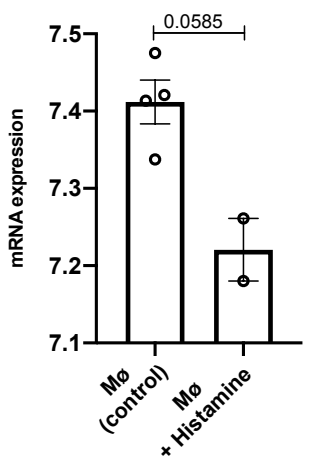

TTC17

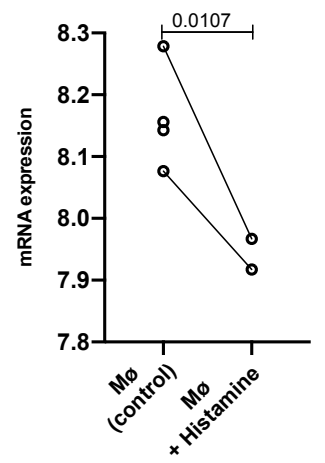

SPC25

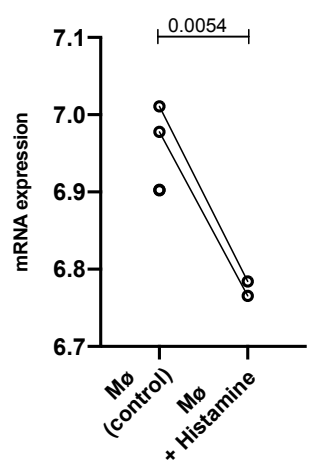

SRFBP1

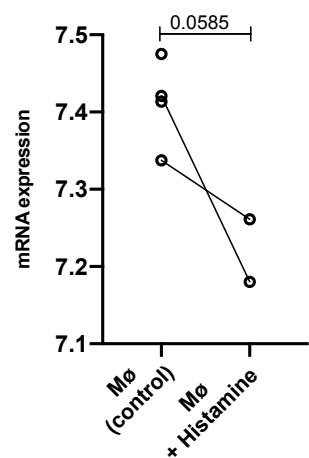

Figure 21: The Cytoskeleton: Genes involved in cytoskeletal dynamics are affected by histamine treatment in macrophages

The expression of the indicated genes is graphically represented by box and whisker plot from minimum to maximum value (left), by bar graph (middle) with the error bars denoting the standard error of the mean, and by symbols and connecting lines (right). For control samples, the two connecting samples on the right hand panel represent macrophages from Donor $A$ and Donor B with and without histamine treatment. There are two additional control samples, unconnected, representing mock treatment and mock histamine treatment. A statistical test was performed to determine if the difference in mRNA expression values between control and histamine-treated samples was significant; the result of this t-test is shown on each graph as a p-value. 

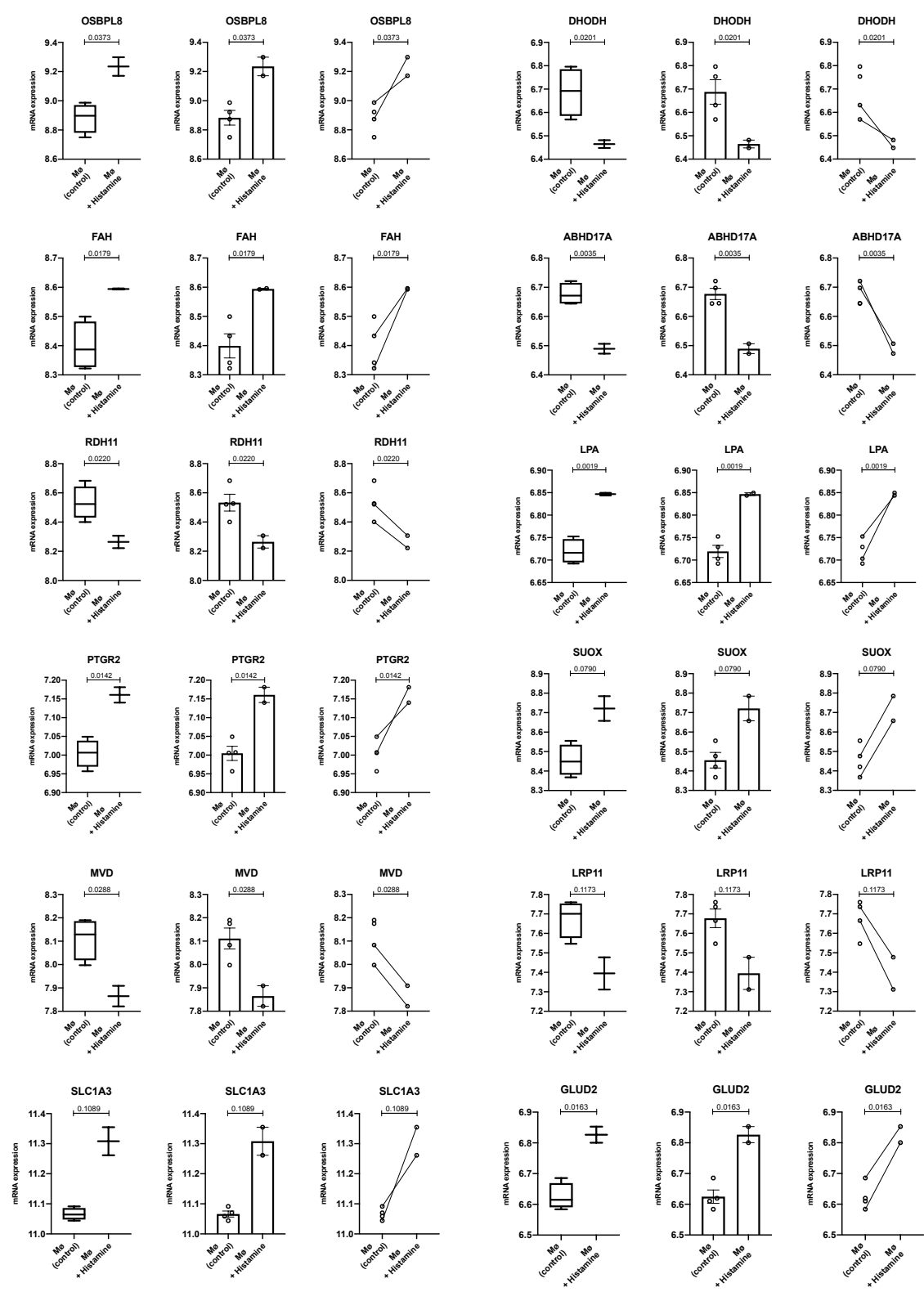

Figure 22: Metabolism: A network of genes that function in cellular metabolism, including genes involved in glutamate metabolism, are differentially expressed following histamine treatment of macrophages

The expression of the indicated genes is graphically represented by box and whisker plot from minimum to maximum value (left), by bar graph (middle) with the error bars denoting the standard error of the mean, and by symbols and connecting lines (right). For control samples, the two connecting samples on the right hand panel represent macrophages from Donor $A$ and Donor B with and without histamine treatment. There are two additional control samples, unconnected, representing mock treatment and mock histamine treatment. A statistical test was performed to determine if the difference in mRNA expression values between control and histamine-treated samples was significant; the result of this t-test is shown on each graph as a p-value. 

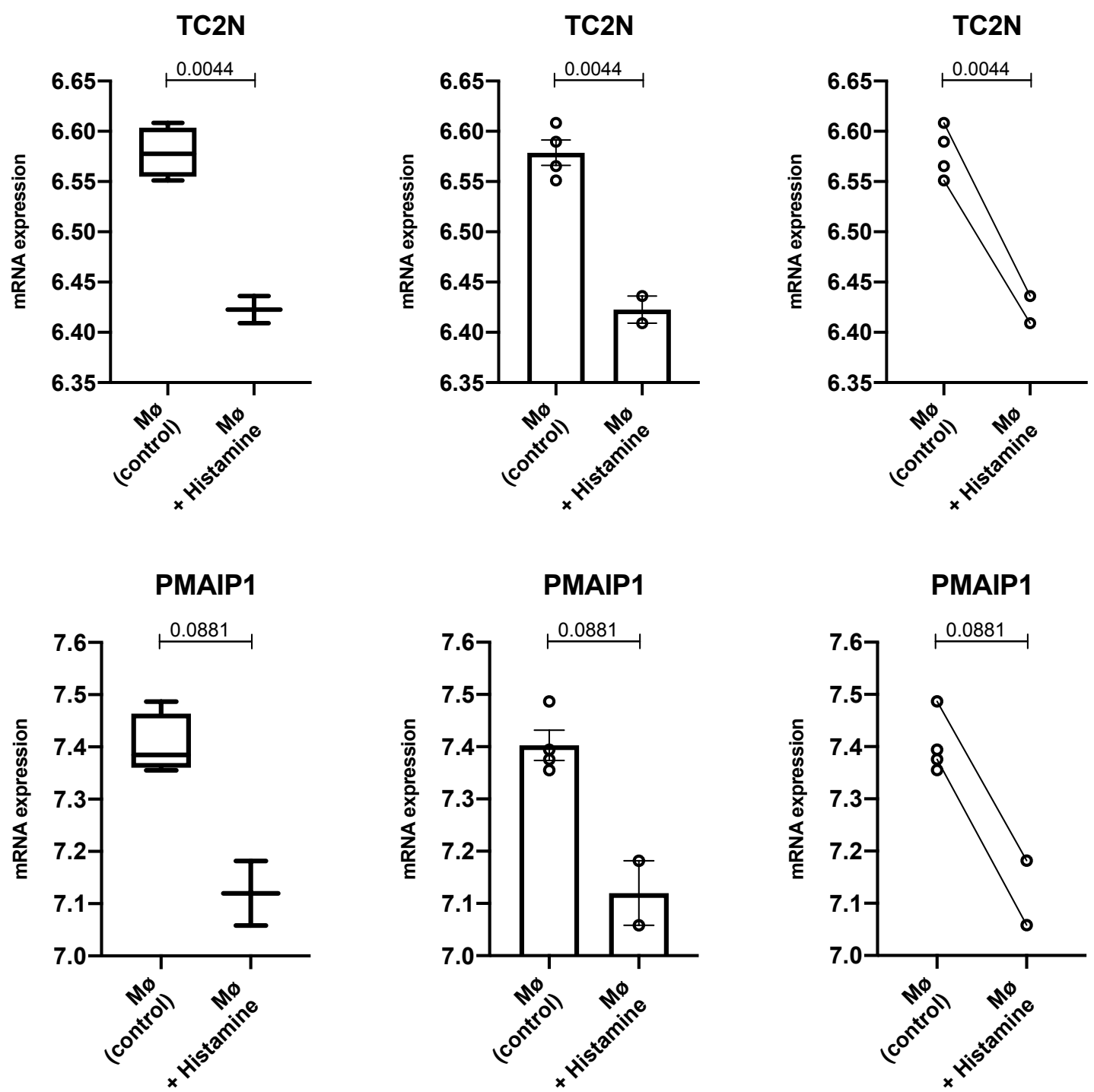

Figure 23: Survival: NOXA (PMAIP1) and TC2N gene expression is differentially expressed by macrophages following histamine exposure.

The expression of the indicated genes is graphically represented by box and whisker plot from minimum to maximum value (left), by bar graph (middle) with the error bars denoting the standard error of the mean, and by symbols and connecting lines (right). For control samples, the two connecting samples on the right hand panel represent macrophages from Donor $A$ and Donor B with and without histamine treatment. There are two additional control samples, unconnected, representing mock treatment and mock histamine treatment. A statistical test was performed to determine if the difference in mRNA expression values between control and histamine-treated samples was significant; the result of this t-test is shown on each graph as a p-value. 
KRTAP10-12

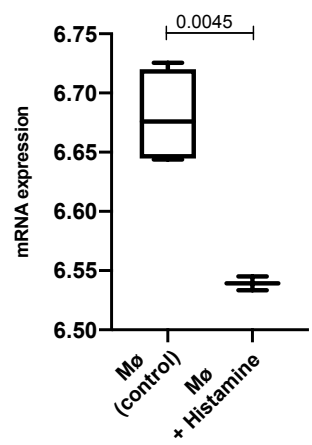

KRT39
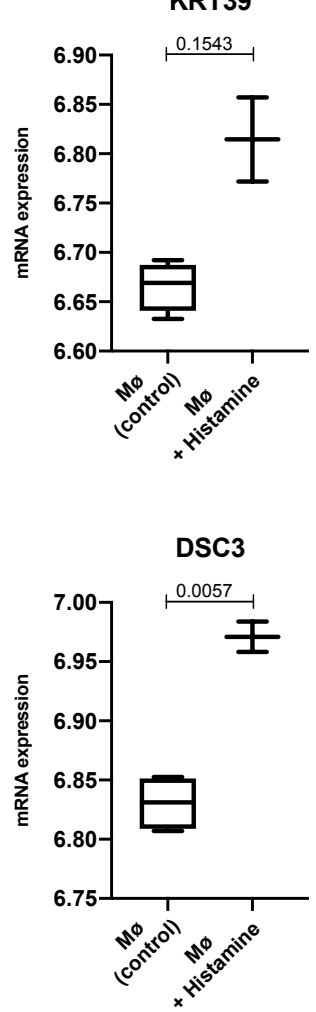

KRTAP10-12

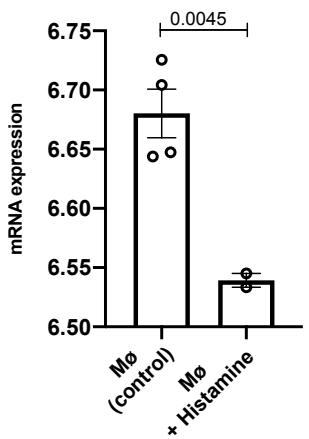

KRT39
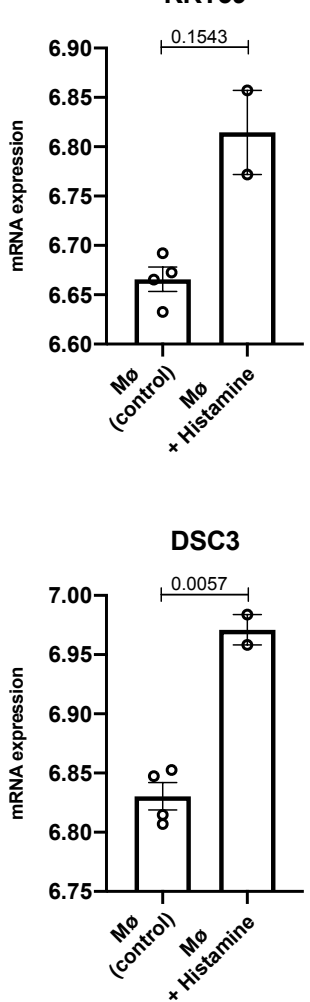

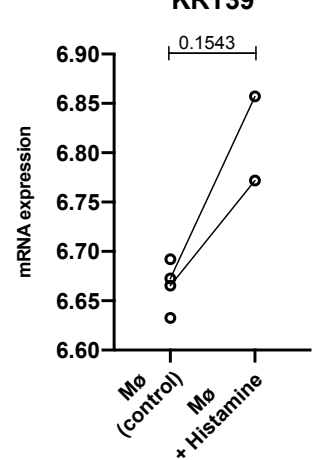

KRTAP10-12

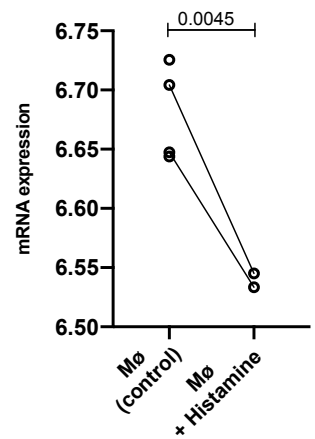

KRT39

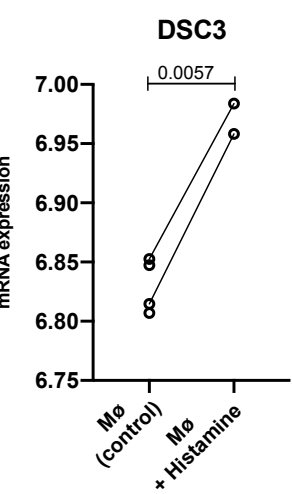

Figure 24: The Skin: KRT39, KRTAP10-12, and DSC3 gene expression is significantly affected by histamine exposure on a transcriptome-wide level.

The expression of the indicated genes is graphically represented by box and whisker plot from minimum to maximum value (left), by bar graph (middle) with the error bars denoting the standard error of the mean, and by symbols and connecting lines (right). For control samples, the two connecting samples on the right hand panel represent macrophages from Donor $\mathrm{A}$ and Donor B with and without histamine treatment. There are two additional control samples, unconnected, representing mock treatment and mock histamine treatment. A statistical test was performed to determine if the difference in mRNA expression values between control and histamine-treated samples was significant; the result of this t-test is shown on each graph as a p-value. 


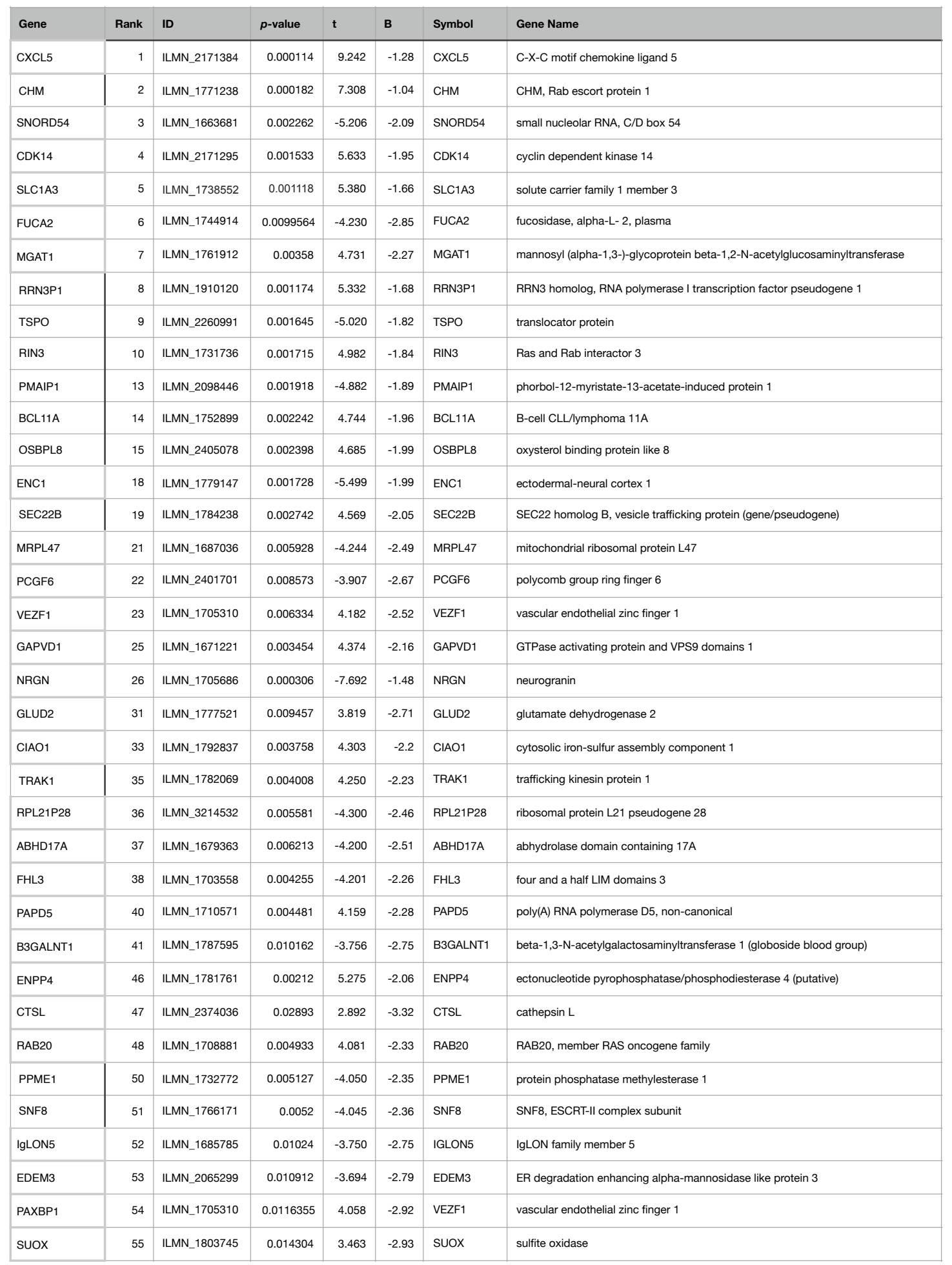

Table 1: The most differentially expressed genes in primary human macrophages following exposure to histamine.

The gene symbol, rank of differential exposure out of 47323 genes, Illumina probe ID, $p$-value with respect to differential expression transcriptome-wide, t statistic, and $B$, the log-odds that the gene is differentially expressed between control and histamine-treated groups, as well as gene name are listed in this chart (all data obtained from GEO2R). 


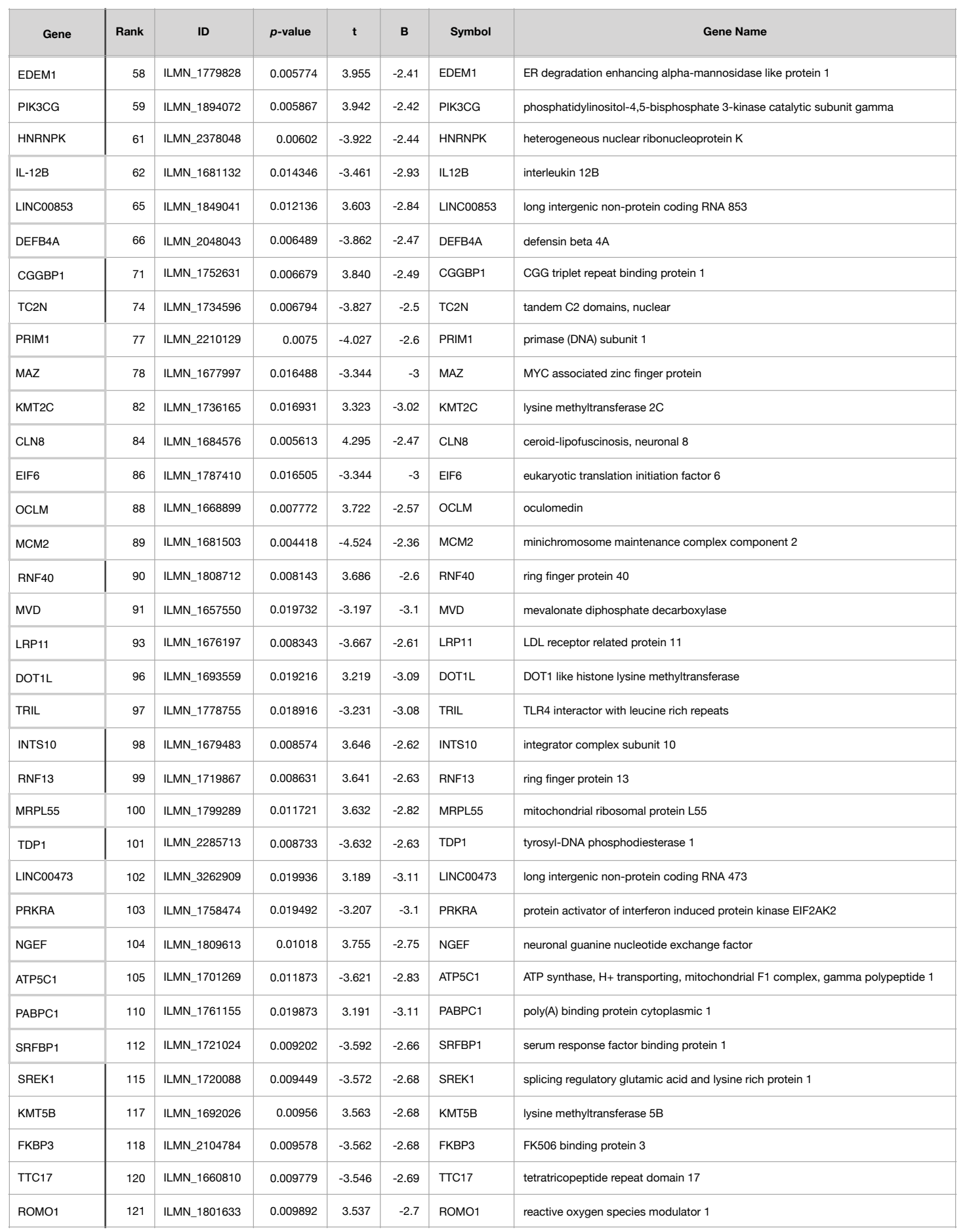

Table 1: The most differentially expressed genes in primary human macrophages following exposure to histamine.

The gene symbol, rank of differential exposure out of 47323 genes, Illumina probe ID, $p$-value with respect to differential expression transcriptome-wide, t statistic, and $B$, the log-odds that the gene is differentially expressed between control and histamine-treated groups, as well as gene name are listed in this chart (all data obtained from GEO2R). 


\begin{tabular}{|c|c|c|c|c|c|c|c|}
\hline Gene & Rank & ID & $p$-value & $\mathbf{t}$ & B & Symbol & Gene Name \\
\hline NUP37 & 123 & ILMN_1771903 & 0.009947 & 3.533 & -2.7 & NUP37 & nucleoporin 37 \\
\hline SPC25 & 124 & ILMN_1814281 & 0.009987 & -3.530 & -2.71 & SPC25 & SPC25, NDC80 kinetochore complex component \\
\hline APEX2 & 125 & ILMN_1652505 & 0.010012 & 3.528 & -2.71 & APEX2 & apurinic/apyrimidinic endodeoxyribonuclease 2 \\
\hline ABL2 & 126 & ILMN_1657870 & 0.010594 & 3.720 & -2.77 & ABL2 & ABL proto-oncogene 2 , non-receptor tyrosine kinase \\
\hline PTGR2 & 128 & ILMN_2105253 & 0.010254 & 3.510 & -2.72 & PTGR2 & prostaglandin reductase 2 \\
\hline DSC3 & 130 & ILMN_2359159 & 0.010533 & 3.490 & -2.74 & DSC3 & desmocollin 3 \\
\hline WNK1 & 131 & ILMN_1788711 & 0.010595 & 3.485 & -2.74 & WNK1 & WNK lysine deficient protein kinase 1 \\
\hline USP4 & 136 & ILMN_1798712 & 0.010741 & 3.475 & -2.75 & USP4 & ubiquitin specific peptidase 4 \\
\hline PCSK1N & 137 & ILMN_1755582 & 0.010749 & 3.475 & -2.75 & PCSK1N & proprotein convertase subtilisin/kexin type 1 inhibitor \\
\hline VAMP7 & 139 & ILMN_1690524 & 0.011184 & -3.445 & -2.77 & VAMP7 & vesicle associated membrane protein 7 \\
\hline JOSD1 & 140 & ILMN_1668535 & 0.011204 & 3.444 & -2.77 & JOSD1 & Josephin domain containing 1 \\
\hline RAB6B & 142 & ILMN_1752299 & 0.011439 & 3.428 & -2.78 & RAB6B & RAB6B, member RAS oncogene family \\
\hline ST13P4 & 143 & ILMN_1726308 & 0.018147 & -3.265 & -3.06 & ST13P4 & $\begin{array}{l}\text { suppression of tumorigenicity } 13 \text { (colon carcinoma) (Hsp70 interacting protein) } \\
\text { pseudogene } 4\end{array}$ \\
\hline RDH11 & 144 & ILMN_2128741 & 0.012096 & -3.605 & -2.84 & RDH11 & retinol dehydrogenase 11 (all-trans/9-cis/11-cis) \\
\hline TRIM49D1 & 146 & ILMN_1681848 & 0.011904 & 3.398 & -2.8 & TRIM49D1 & tripartite motif containing 49D1 \\
\hline UGGT2 & 150 & ILMN_1788387 & 0.012016 & -3.391 & -2.81 & UGGT2 & UDP-glucose glycoprotein glucosyltransferase 2 \\
\hline POLR3H & 153 & ILMN_1786024 & 0.012151 & -3.383 & -2.82 & POLR3H & RNA polymerase III subunit $\mathrm{H}$ \\
\hline HNRNPCL1 & 154 & ILMN_2154186 & 0.012178 & 3.381 & -2.82 & HNRNPCL1 & heterogeneous nuclear ribonucleoprotein C-like 1 \\
\hline ADAT2 & 156 & ILMN_3245693 & 0.018456 & 3.252 & -3.07 & ADAT2 & adenosine deaminase, tRNA specific 2 \\
\hline $\mathrm{CCNH}$ & 157 & ILMN_1742250 & 0.012397 & 3.368 & -2.83 & $\mathrm{CCNH}$ & cyclin $\mathrm{H}$ \\
\hline PDIA3P1 & 158 & ILMN_2075436 & 0.012498 & -3.362 & -2.83 & PDIA3P1 & protein disulfide isomerase family A member 3 pseudogene 1 \\
\hline PCDH11Y & 161 & ILMN_2254722 & 0.012565 & 3.358 & -2.84 & PCDH11Y & protocadherin 11 Y-linked \\
\hline TRIM14 & 163 & ILMN_1713542 & 0.012612 & 3.355 & -2.84 & TRIM14 & tripartite motif containing 14 \\
\hline TCF12 & 164 & ILMN_1669832 & 0.012627 & 3.354 & -2.84 & TCF12 & transcription factor 12 \\
\hline GALNT16 & 167 & ILMN_1799903 & 0.012834 & -3.342 & -2.85 & GALNT16 & polypeptide $\mathrm{N}$-acetylgalactosaminyltransferase 16 \\
\hline SRL & 168 & ILMN_1736692 & 0.012902 & 3.338 & -2.85 & SRL & sarcalumenin \\
\hline KRT39 & 176 & ILMN_1760665 & 0.013306 & 3.315 & -2.87 & KRT39 & keratin 39 \\
\hline PPP2R3A & 177 & ILMN_1702708 & 0.011847 & 3.623 & -2.83 & PPP2R3A & protein phosphatase 2 regulatory subunit B"alpha \\
\hline NOX1 & 179 & ILMN_1778077 & 0.018344 & -3.257 & -3.06 & NOX1 & NADPH oxidase 1 \\
\hline SYNE1 & 180 & ILMN_1666785 & 0.013414 & -3.310 & -2.87 & SYNE1 & spectrin repeat containing nuclear envelope protein 1 \\
\hline OLA1 & 182 & ILMN_1709075 & 0.010582 & 3.721 & -2.77 & OLA1 & Obg-like ATPase 1 \\
\hline WDFY1 & 183 & ILMN_1676448 & 0.013491 & 3.305 & -2.88 & WDFY1 & WD repeat and FYVE domain containing 1 \\
\hline $\operatorname{miR} 1470$ & 184 & ILMN_3308290 & 0.018779 & -3.237 & -3.08 & MIR1470 & microRNA 1470 \\
\hline CALD1 & 186 & ILMN_2324002 & 0.013846 & -3.286 & -2.89 & CALD1 & caldesmon 1 \\
\hline KTN1 & 187 & ILMN_2339202 & 0.013949 & -3.28066 & -2.89 & KTN1 & kinectin 1 \\
\hline LFNG & 189 & ILMN_1663080 & 0.014179 & 3.269 & -2.9 & LFNG & LFNG O-fucosylpeptide 3-beta-N-acetylglucosaminyltransferase \\
\hline LGALS9C & 190 & ILMN_1766184 & 0.014196 & -3.268 & -2.9 & LGALS9C & galectin 9C \\
\hline
\end{tabular}

Table 1: The most differentially expressed genes in primary human macrophages following exposure to histamine.

The gene symbol, rank of differential exposure out of 47323 genes, Illumina probe ID, $p$-value with respect to differential expression transcriptome-wide, t statistic, and $B$, the log-odds that the gene is differentially expressed between control and histamine-treated groups, as well as gene name are listed in this chart (all data obtained from GEO2R). 


\begin{tabular}{|c|c|c|c|c|c|c|c|}
\hline CASR & 194 & ILMN_1767711 & 0.014501 & -3.252 & -2.92 & CASR & calcium sensing receptor \\
\hline GDPD1 & 196 & ILMN_1729599 & 0.014693 & 3.242 & -2.92 & GDPD1 & glycerophosphodiester phosphodiesterase domain containing 1 \\
\hline UAP1L1 & 197 & ILMN_1653712 & 0.014881 & 3.233 & -2.93 & UAP1L1 & UDP-N-acetylglucosamine pyrophosphorylase 1 like 1 \\
\hline $\mathrm{FAH}$ & 203 & ILMN_1781536 & 0.005075 & 4.390 & -2.42 & $\mathrm{FAH}$ & fumarylacetoacetate hydrolase \\
\hline PLPP3 & 208 & ILMN_2388800 & 0.015307 & 3.212 & -2.95 & PLPP3 & phospholipid phosphatase 3 \\
\hline cox7B & 212 & ILMN_2184049 & 0.017049 & -3.317 & -3.02 & cox7B & cytochrome $\mathrm{c}$ oxidase subunit 7B \\
\hline POLR2F & 214 & ILMN_1745885 & 0.015583 & 3.199 & -2.96 & POLR2F & RNA polymerase II subunit $F$ \\
\hline KRTAP10-12 & 221 & ILMN_1754009 & 0.016108 & -3.175 & -2.98 & KRTAP10-12 & keratin associated protein 10-12 \\
\hline BTBD1 & 222 & ILMN_2411264 & 0.016189 & -3.172 & -2.98 & BTBD1 & BTB domain containing 1 \\
\hline SUCLG1 & 224 & ILMN_1779616 & 0.016339 & -3.165 & -2.99 & SUCLG1 & succinate-CoA ligase alpha subunit \\
\hline MIR192 & 226 & ILMN_3308906 & 0.01642 & 3.161 & -2.99 & MIR192 & microRNA 192 \\
\hline PIP4K2C & 228 & ILMN_1787308 & 0.01652 & 3.157 & -2.99 & PIP4K2C & phosphatidylinositol-5-phosphate 4-kinase type 2 gamma \\
\hline C1QTNF3 & 229 & ILMN_1768925 & 0.016968 & -3.321 & -3.02 & C1QTNF3 & C1q and tumor necrosis factor related protein 3 \\
\hline STGGALNAC3 & 230 & ILMN_1779061 & 0.016569 & 3.15470 & -2.99 & ST6GALNAC3 & ST6 N-acetylgalactosaminide alpha-2,6-sialyltransferase 3 \\
\hline MIR202 & 232 & ILMN_3310920 & 0.016609 & -3.153 & -3 & MIR202 & microRNA 202 \\
\hline UTP14C & 238 & ILMN_1686645 & 0.0169 & 3.140 & -3.01 & UTP14C & UTP14, small subunit processome component homolog C (S. cerevisiae) \\
\hline HPS3 & 239 & ILMN_1762224 & 0.016941 & 3.139 & -3.01 & HPS3 & HPS3, biogenesis of lysosomal organelles complex 2 subunit 1 \\
\hline LPA & 241 & ILMN_1766590 & 0.017156 & 3.129 & -3.01 & LPA & lipoprotein(a) \\
\hline DHODH & 247 & ILMN_1782993 & 0.017501 & -3.115 & -3.03 & DHODH & dihydroorotate dehydrogenase (quinone) \\
\hline LMNA & 249 & ILMN_1696749 & 0.017635 & 3.109 & -3.03 & LMNA & $\operatorname{lamin} \mathrm{A} / \mathrm{C}$ \\
\hline
\end{tabular}

\section{Table 1: The most differentially expressed genes in primary human macrophages following exposure to histamine.}

The gene symbol, rank of differential exposure out of 47323 genes, Illumina probe ID, $p$-value with respect to differential expression transcriptome-wide, t statistic, and $B$, the log-odds that the gene is differentially expressed between control and histamine-treated groups, as well as gene name are listed in this chart (all data obtained from GEO2R). 